\title{
An Alternative to the Alcubierre Theory: Warp Fields by the Gravitation via Accelerated Particles Assertion
}

\author{
Edward A. Walker ${ }^{1}$ \\ ${ }^{1}$ Mathematics Department, Miami Gardens, Florida Memorial University, United States \\ Correspondence: Edward A. Walker, Mathematics Department, Miami Gardens, Florida Memorial University, \\ United States. E-mail: dwrdwalker@yahoo.com
}

Received: September 6, 2016

Accepted: September 21, 2016

Online Published: September 30, 2016

doi:10.5539/apr.v8n5p44

URL: http://dx.doi.org/10.5539/apr.v8n5p44

\begin{abstract}
A summarization of the Alcubierre metric is given in comparison to a new metric that has been formulated based on the theoretical assertion of a recently published paper entitled "gravitational space-time curve generation via accelerated particles". The new metric mathematically describes a warp field where particle accelerators can theoretically generate gravitational space-time curves that compress or contract a volume of space-time toward a hypothetical vehicle traveling at a sub-light velocity contingent upon the amount of voltage generated. Einstein's field equations are derived based on the new metric to show its compatibility to general relativity. The "time slowing" effects of relativistic gravitational time dilation inherent to the gravitational field generated by the particle accelerators is mathematically shown to be counteracted by a gravitational equilibrium point between an arrangement of two equal magnitude particle accelerators. The gravitational equilibrium point produces a volume of flat or linear space-time to which the hypothetical vehicle can traverse the region of contracted space-time without experiencing time slippage. The theoretical warp field possessing these attributes is referred to as the two gravity source warp field which is mathematically described by the new metric.
\end{abstract}

Keywords: gravitation, particle accelerator, space-time metric, Einstein's field equations, Alcubierre warp field, gravitational time dilation, space-time compression

\section{Introduction}

A theoretical warp field and therefore a space-time metric is mathematically formulated based on the theoretical notion of the generation of gravitational space-time curves produced by massive charged particles accelerated to the precipice of the speed of light introduced by the Gravitational space-time curve generation via accelerated particles paper (Walker, 2016). Resultantly, It is asserted that particle accelerators with sufficient voltage values can generate a gravitational field and its corresponding space-time curve (Walker, 2016). The new theoretical warp field based on this assertion is referred to as the two gravity source warp field and is compared to the famous Alcubierre warp field theory.

Thus, the Alcubierre warp field assertion is summarized in comparison to the theoretical assertion of the two gravity source warp field in section 1 . The velocity based gravitation by accelerated particles metric (VBGAP metric) which is used to described the two gravity source warp field is introduced and mathematically formulated based on the assertion of the Gravitational space-time curve generation via accelerated particles paper (Walker, 2016). The VBGAP metric describing the two gravity source warp field is formulated in accordance to Einstein's field equations. Therefore, Einstein's field equations are derived based on the VBGAP metric to show that the VBGAP metric is valid to general relativity.

An arrangement of two particle accelerators generating two gravitational fields in close proximity to one another is theoretically shown to have the ability to compensate for relativistic gravitational time dilation within the gravitational fields and whose combined gravitational field can warp or contract space-time toward a hypothetical vehicle as it moves spatially with a sub light velocity (hence the two gravity source warp field). Therefore, the issue of gravitational time dilation resulting from the generation of the gravitational fields is mathematically shown to be resolved by an arrangement of two particle accelerators producing a gravitational equilibrium point. The notion of the two gravity source warp field is then mathematically incorporated into the VBGAP metric describing the motion of a hypothetical vehicle. The mathematical incorporation of the two 
gravity source warp field concept into the VBGAP metric allows the derivation of mathematical equations used to obtain hypothetical quantitative results and allow conclusive thoughts on the study.

\section{The Alcubierre Warp Field vs. the Two Gravity Source Warp Field}

In 1994, physicist Miguel Alcubierre formulated a space-time metric which is a unique solution to Einstein's field equations (Anderson, 2016). The Alcubierre metric describes a warp field to which space-time is compressed or contracted towards a hypothetical vehicle and expanded behind it (Anderson, 2016). In general relativity and/or cosmology the universe expands theoretically creating new space with gravity conversely having the ability to contract or compress it, the Alcubierre metric suggest that a similar phenomenon can transpire in a local sense (Cramer, 1996). This gives to the implication that both the expansion and contraction of space-time could be localized to a vehicle. The possibility for faster than light travel (FTL) in the Alcubierre theory comes from the theoretical ability to compress and expand space-time faster than the speed of light, which is permissible by general relativity (Cramer, 1996). Hence, this gives the possibility of contracting and expanding space-time to allow a vehicle within the region of warped space-time to cross distances in what equates to super luminous velocities relatively (Anderson, 2016). The area of warped space-time is referred to as a warp bubble (Anderson, 2016), within this warp bubble, the hypothetical vehicle travels along a world line or a geodesic embedded on a space-time manifold as required by Einstein's field equations of general relativity. A more detailed description of Alcubierre's warp concept is that it is constructed of hyperbolic tangent functions which create a very peculiar distortion of space at the edges of the flat-space volume (Cramer, 1996). The metric corresponding to this description (or the Alcubiere metric) is expressed such that (Anderson, 2016):

$$
d s^{2}=\left(v_{s}(t)^{2} f\left(r_{s}(t)\right)^{2}-1\right) d t^{2}-2 v_{s}(t) f\left(r_{s}(t)\right) d x d t+d x^{2}+d y^{2}+d z^{2}
$$

Where $v_{s}(t)$ is a velocity function (Anderson, 2016), the function $f\left(r_{s}(t)\right)$ is the function of the hyperbolic tangent functions describing the warped geometry of space-time encompassing the hypothetical vehicle (Anderson, 2016). Function $f\left(r_{s}(t)\right)$ is expressed such that (Anderson, 2016):

$$
f\left(r_{S}(t)\right)=\frac{\tanh \left(\sigma\left(r_{S}+R\right)\right)-\tanh \left(\sigma\left(r_{s}-R\right)\right)}{2 \tanh (\sigma R)}
$$

Where $R$ and $\sigma$ are arbitrary parameters $(R>0, \sigma>0)$ (Anderson, 2016). An important fact is that the hypothetical vehicle is not actually in motion but is interpreted by the Alcubierre metric to be in a state of "free fall" along a geodesic on the surface of the space-time manifold to which the warp bubble is defined on (Cramer, 1996). Hence, in a local sense, the vehicle is not actually moving at super luminous velocities (Anderson, 2016). An additional and important consideration is that the hypothetical vehicle at the center of the warp bubble experiences no relativistic mass increase or time dilation as specified by the Alcubierre theory (Cramer, 1996). However, there are issues prohibiting the Alcubierre theory from becoming a theoretical possibility. The first issue is that Alcubierre formulates a metric and applies it to Einstein's field equations (namely the Einstein tensor), mathematically the corresponding momentum-stress-energy tensor requires the generation of a form of exotic matter to whom cannot currently be produced with current technology (Anderson, 2016). Moreover, another issue is that, according to Serguei Krasnikov, it would be impossible to generate the bubble without being able to force the exotic matter to move at faster than light speeds locally, which would require the existence of theoretical tachyons (Anderson, 2016). Lastly, general relativity provides a procedure for determining how much energy density (energy per unit volume) is implicit in a given metric (Cramer, 1996), this suggested the requirement of the generation of high amounts of negative energy which is prevalent in vacuum energy (illustrated by the Casmir effect) on a cosmic scale but cannot be produced in vast amounts required to generate the Alcubierre warp bubble (Cramer, 1996).

A paper published entitled "Gravitational space-time curve generation via accelerated particles" (Walker, 2016) introduced a group of equations describing the theoretical generation of a gravitational space-time curve (and thus gravitational force) by particles accelerated to an approximate 99 percent of the speed of light. An acceleration equal to multiples of the speed of light greater than 1 per unit time is enacted on the particles whose velocities are at an approximate 99 percent of the speed of light (which in reality asymptotically approaches but never achieves the speed of light, thus .99 is simply an approximation for calculations). Resultantly, space-time (and therefore gravitational force) treats or reacts to the particles as if they are more massive. Therefore, the force of gravity and hence the curvature of space-time are proportional to an acceleration or force value which is multiples of the speed of light per unit time greater than 1 . This implies that particle accelerators can potentially generate gravitational fields and space-time curves. This gives way to the formulation of a space-time metric in 
accordance to Einstein's field equations based on the theoretical notion of the gravitational space-time curves generated by accelerated particles assertion. Therefore, a space-time metric is formulated and proposed where a system or hypothetical vehicle produces a gravitational field via particle accelerators which can compress or contract space-time toward the vehicle. A difference to the metric proposed in this paper as compared to the Alcubierre metric is that the vehicle must have a velocity (a sub light velocity), essentially as the hypothetical vehicle travels through space-time at an arbitrary velocity, the generated gravitational fields contract or brings space-time closer to the vehicle permitting it to travel distances faster than it would in flat or linear space at the same velocity. In relating this to general relativity, the hypothetical vehicle is shown to travel on along a geodesic defined by the new metric. Due to the fact that a velocity is included to the gravitational space-time metric produced by the accelerated charged particles, the term "velocity based gravitation by accelerated particles" metric (VBGAP metric) is applied to the metric. However, gravitational time dilation is an issue to the system or vehicle as it transits space-time. The vehicle or system will travel slower through time as opposed to flat or linear space counteracting the spatial distance traveled by the vehicle as space-time is contracted toward the vehicle.

An arrangement of two particle accelerators generating two theoretical gravitational fields will generate an equilibrium point between them which will be shown to generate an area of flat or linear space-time (see section 4 page 19). This equilibrium point between the two particle accelerators will compensate for the time slippage induced by gravitational time dilation. Resultantly, as the hypothetical vehicle travels through space-time at a sub light velocity, the combined effect of the gravitational fields generated by the two particle accelerators compress space-time toward the vehicle permitting spatial distance to be traversed without time slippage due to gravitational time dilation within the two gravitational fields. The gravitational field curving space-time around the system or vehicle generated by the two particle accelerators is referred to as the "two-gravity source" warp field as stated in the introduction. The VBGAP metric is then redefined in terms of the two-gravity source warp field. As compared to the Alcubierre metric, the generation of the warp field is contingent on the electrical energy(or voltage) powering the particle accelerators as opposed to the excessive amounts of negative energy and exotic matter. The two-gravity source warp field described by the VBGAP metric can be in theory generated using technology that is currently available. However, the issue hindering the practicality of the two-gravity source warp field is that exorbitant amounts of energy have to be expended to generate a warp field sufficient to have a substantial affect as shown by the quantitative examples in the conclusion. Lastly, the Alcubierre Warp field expands space-time in the direction opposite the direction of travel of the hypothetical vehicle where two-gravity source warp field contracts space-time in all directions, this issue is addressed in section 5.

\section{The Velocity Based Gravitation by Accelerated Particles Metric (VBGAP Metric)}

Section 2 introduces and formulates the velocity based gravitation by accelerated particles metric or the VBGAP metric. As aforementioned, this warp concept is based on the theoretical notion introduced in the "Gravitational space-time curve generation via accelerated particles" paper (Walker, 2016). Therefore, as presented in the "Gravitational space-time curve generation via accelerated particles" paper (Walker, 2016), a force value $F_{a}$ is generated on a total mass value $m_{p}$ (the mass or combined mass of charged particles) by an electromagnetic particle accelerator. Force $F_{a}$ is stated such that (Young \& Freedman, 2004; Walker, 2016):

$$
F_{a}=a_{c} m_{p}=\left(\frac{N_{a} c}{\Delta t}\right) m_{p}, \quad N_{a} \in R, \quad N_{a} \geq 1
$$

The acceleration value $a_{c}$ has a value $a_{c}=N_{a} c / \Delta t$ in Newton's equation of force ( $\mathrm{F}=\mathrm{ma}$ ) above (Young \& Freedman, 2004; Walker, 2016). The speed of light is denoted $c$ and variable $N_{a}$ (and its conditions expressed above) is referred to as the particle acceleration number. Thus momentum value $p_{a}$ is the integral of force value $F_{a}$ with respect to time $t$ as shown below (Young \& Freedman, 2004; Walker, 2016).

$$
p_{a}=\int_{0}^{\Delta t} F_{a} d t=N_{a} c m_{p}
$$

Note, the particles of mass $m_{p}$ do not achieve super luminous velocities as dictated by relativity (Young \& Freedman, 2004). A momentum value $p_{c}$ is given such that (Walker, 2016):

$$
p_{c}=(.99)(c) m^{\prime}
$$

The particles' actual velocity is stated to be an approximated 99 percent of the speed of light $(.99 c<c)$ as shown above. Mass value $m^{\prime}$ is stated to be the "variable inertial mass" (Walker, 2016) which will be referred to as variable mass in short further on. Momentum value $p_{a}$ is set equal to momentum value $p_{c}$ as shown below (Walker, 2016). 
This can be expressed such that:

$$
p_{c}=p_{a}
$$

$$
N_{a} c m_{p}=(.99)(c) m^{\prime}
$$

Solving equation 2.04 for variable inertial mass $m^{\prime}$ gives (Walker, 2016):

$$
m^{\prime}=\frac{N_{a} m_{p}}{.99}
$$

As derived in the "Gravitational space-time curve generation via accelerated particles" paper (Walker, 2016), this equation (2.05) can be stated as the "Variable mass increase equation". The actual interpretation of the variable mass equation that was not directly elucidated in the "Gravitational space-time curve generation via accelerated particles" paper is that variable mass $m^{\prime}$ is not a literal increase in inertial mass rather it is a description of how space-time treats or reacts to mass when it is accelerated to the verge of the speed of light having an acceleration value that is multiples greater than the speed of light acting on it (acceleration $a_{c}$ of equation 2.0). Thus as acceleration number $N_{a}$ increases to multiples greater than the speed of light $c$, force value $F_{a}$ (and therefore acceleration $a_{c}$ of equation 2.0) and momentum value $p_{a}$ increase (Walker, 2016). However, the value of the particles' velocity remains below the speed of light as indicated by the equivalence to momentum value $p_{c}\left(p_{c}=(.99)(c) m^{\prime}\right)($ equation 2.03$)$, resultantly variable inertial mass $m^{\prime}$ must mathematically increase as acceleration number $N_{a}$ increases (Walker, 2016).

To avoid confusion (as stated in the "Gravitational space-time curve generation via accelerated particles" paper (Walker, 2016), it must be noted that relativistic mass dilation is different from the variation of variable mass or inertial $m^{\prime}$ as the particle approaches the speed of light. This can be conveyed by setting variable inertial mass $m^{\prime}$ equal to the product of the variable inertial mass and the Lorentz factor $\gamma$ as shown below (Young \& Freedman, 2004; Walker, 2016).

$$
m^{\prime}=m^{\prime} \gamma
$$

This can alternatively be expressed such that (Young \& Freedman, 2004; Walker, 2016):

$$
m^{\prime}=m^{\prime} \boldsymbol{\gamma}=m^{\prime}\left[1-\frac{v^{2}}{c^{2}}\right]^{-1 / 2}
$$

Equation 2.07 implies that the Lorentz factor $\gamma$ is equal to 1 and implies that relative velocity $v$ or the velocity of an observer is zero $(v=0)$ for the purpose of this derivation (Young \& Freedman, 2004; Walker, 2016).

$$
m^{\prime}=m^{\prime}\left[1-\frac{(0)^{2}}{c^{2}}\right]^{-1 / 2}
$$

Conclusively, inertial mass $m^{\prime}$ does not vary according to the Lorentz factor $\gamma$ (Walker, 2016). In continuing the derivation of the VBGAP metric, a gravitational field $F_{g}^{\prime}$ between variable mass $m^{\prime}$ and a test particle of mass $m_{0}$ separated by distance $r$ is expressed such that (Young \& Freedman, 2004; Walker, 2016):

$$
F_{g}^{\prime}=\frac{G m^{\prime} m_{0}}{r^{2}}
$$

This can alternatively be expressed such that (Walker, 2016):

$$
F_{g}^{\prime}=\frac{G\left(N_{a} m_{p}\right) m_{0}}{r^{2}(.99)}, \quad N_{a} \in R, \quad N_{a} \geq 1
$$

Gravitational force $F_{g}^{\prime}$ varies according to variable mass $m^{\prime}$ which varies according to acceleration number $N_{a}$ (Walker, 2016). In the interest of applying this mathematical concept to the application of real world particle accelerators, the voltage $V_{a}$ required to accelerate the particles to produce a gravitational field $F_{g}^{\prime}$ has been formulated using the Lorentz force equation in the "Gravitational space-time curve generation via accelerated particles" paper (Walker, 2016) as shown below (see appendix A for the formulation of this equation) (Walker, 2016).

$$
V_{a}=-c\left[\left(\frac{m_{p} N_{a}}{q \Delta t}\right)^{2}-((.99) B)^{2}\right]^{\frac{1}{2}} ; \quad\left(\frac{m_{p} N_{a}}{q \Delta t}\right)^{2}>\quad((.99) B)^{2}
$$

Where $N_{a}$ is the acceleration number, $m_{p}$ is the mass of the particles being accelerated, $c$ is the speed of light, $q$ is the charge of the particle(s), $B$ is the value of the associated magnetic field, and $\Delta t$ is the interval of time (Walker, 2016). 
Gravitational potential energy is given by the integral of gravitational force $F_{g}^{\prime}$ of equation 2.10 in respect to distance $r$ (Young \& Freedman, 2004; Walker, 2016).

$$
U_{g}^{\prime}=\int F_{g}^{\prime} d r=\frac{G\left(N_{a} m_{p}\right) m_{0}}{r(.99)}
$$

Distance $r$ is substituted for the Schwarzchild radius $r_{s}\left(r=r_{s}\right)$, therefore equation 2.12 can be re-expressed such that (Young \& Freedman, 2004; Walker, 2016):

$$
U_{g}^{\prime}=\int F_{g}^{\prime} d r=\frac{G\left(N_{a} m_{p}\right) m_{0}}{r_{s}(.99)}
$$

Potential energy $U_{g}^{\prime}$ is set equal to the maximum value of kinetic energy of mass $m_{0}$ at the speed of light $c$ denoted $K_{\max }$ (Young \& Freedman, 2004; Walker, 2016).

$$
K_{\max }=\frac{m_{0} c^{2}}{2}=U_{g}^{\prime}
$$

This equivalence can be stated such that (Young \& Freedman, 2004; Walker, 2016):

$$
\frac{m_{0} c^{2}}{2}=\frac{G\left(N_{a} m_{p}\right) m_{0}}{r_{S}(.99)}
$$

Solving equation 2.15 for variable $r_{s}$ gives the value of the Schwarzchild radius such that (Wald, 1984; Young \& Freedman, 2004; Walker, 2016):

$$
r_{s}=\frac{2 G\left(N_{a} m_{p}\right)}{(.99) c^{2}}
$$

It is of great importance in formulating the VBGAP metric to state that the entire system being described has a sub-light velocity value of $v_{s}\left(v_{s}<c\right)$. Where time value $t_{a}$ is a constant or fixed value of time, distance $r_{a}$ is stated such that:

$$
r_{a}=t_{a} v_{s}
$$

Thus radius $r^{\prime}$ is the sum of Schwarzchild radius $r_{s}$ (of equation 2.16) and distance $r_{a}$ such that:

This can be expressed as:

$$
r^{\prime}=r_{a}+r_{s} ; \quad r^{\prime}>r_{s}
$$

$$
r^{\prime}=r_{a}+\frac{2 G\left(N_{a} m_{p}\right)}{(.99) c^{2}}
$$

Therefore, the compression factor denoted $a\left(r_{s}\right)$ has a value and condition such that (Wald, 1984):

$$
a\left(r_{s}\right)=\frac{r^{\prime}-r_{s}}{r^{\prime}}=1-\frac{r_{s}}{r^{\prime}}=1-\frac{2 G\left(N_{a} m_{p}\right)}{r^{\prime}(.99) c^{2}} ; 1>a\left(r_{s}\right)
$$

Compression factor $a\left(r_{s}\right)$ is the compression of the geometry of space due to gravitational force $F_{g}^{\prime}$ produced by the particle accelerator. Therefore, it can be stated that the compression factor $a\left(r_{s}\right)$ contracts space-time. A priori is that the compression or contraction of space-time is measured relative to radius $r^{\prime}$. A displacement $x_{\amalg}^{\prime}$ is expressed such that:

$$
x_{ц}^{\prime}=t v_{S}
$$

Thus displacement $x_{\longleftarrow}^{\prime}$ is defined at system velocity $v_{s}$ at a variable of time $t$. It is important to cite that the variable of time $t$ is different from the constant value of time $t_{a}$ of equation 2.17. Time value $t$ varies while time value $t_{a}$ is a constant parameter set at a value of one second $\left(t_{a}=1 \mathrm{~s}\right)$. Hence, in regards to the operations of differentiation and integration in respect to time value $t$, time value $t_{a}$ is treated as a constant. Time $t_{a}$ is simply a temporal parameter of measurement for the volume of space-time being influenced by the gravitational force of the particle accelerator. Displacement $\Delta x_{\longleftarrow}^{\prime}$ is the product between the displacement $x_{\amalg}^{\prime}$ and the inverse of compression factor $a\left(r_{s}\right)$ and its condition expressed below.

$$
\Delta x_{\amalg}^{\prime}=x_{\amalg}^{\prime}\left(a\left(r_{S}\right)\right)^{-1}=\frac{t v_{S}}{\left(1-\frac{r_{S}}{r^{\prime}}\right)} ; \quad \Delta x_{\amalg}^{\prime}>x_{\amalg}^{\prime}
$$

This can alternatively be expressed as: 


$$
\Delta x_{\amalg}^{\prime}=\left(t v_{s}\right)\left[1-\frac{2 G\left(N_{a} m_{p}\right)}{r^{\prime}(.99) c^{2}}\right]^{-1}
$$

The most obvious question is "why is the displacement $x_{\amalg}^{\prime}$ multiplied to the inverse of compression factor $a\left(r_{s}\right)$ (expressed as $\left.\left(a\left(r_{s}\right)\right)^{-1}\right)$ ?". The displacement $\Delta x_{\amalg}^{\prime}$ of equation 2.23 measures the movement of velocity $v_{s}$ at time $t$ relative to a stationary observer outside of the generated gravitational field which is also beyond the effects (in flat space) of the spatial compression of compression factor $a\left(r_{s}\right)$. Therefore, the Lorentz factor $\gamma$

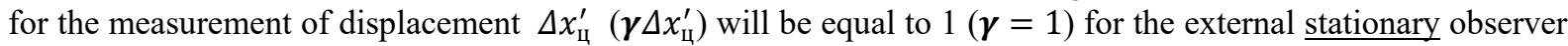
for having a relative velocity of zero $(v=0)$ (Young \& Freedman, 2004). The external stationary observer would perceive the system to be traveling a greater distance over time $t$ as the system transits over compressed space-time and thus spatial distance. Mathematically speaking, as measured by the external stationary observer,

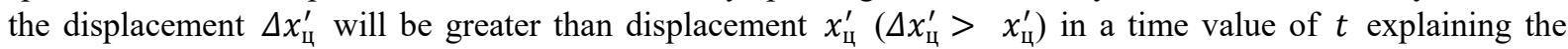
inverse coefficient of $\left(a\left(r_{s}\right)\right)^{-1}$ of the compression factor. An important fact to point out is that as the warped space caused by the gravitational fields generated by the particle accelerator passes through a region space, space flattens or reverts back to its linear form once the system has passed.

The basis of measurement in a four dimensional Minkowski space is defined by $\sigma^{a b}$ which is a collection of four 4-space basis vectors (Wald, 1984).

$$
\sigma^{a b}=\left[\begin{array}{c}
\sigma^{0 b} \\
\sigma^{1 b} \\
\sigma^{2 b} \\
\sigma^{3 b}
\end{array}\right]=\left[\begin{array}{c}
\sigma^{00} \sigma^{01} \sigma^{02} \sigma^{03} \\
\sigma^{10} \sigma^{11} \sigma^{12} \sigma^{13} \\
\sigma^{20} \sigma^{21} \sigma^{22} \sigma^{23} \\
\sigma^{30} \sigma^{31} \sigma^{32} \sigma^{33}
\end{array}\right]
$$

The four 4-space basis vectors of $\sigma^{a b}$ constitute the axis of measurement to an arbitrary external stationary observer geometrically measuring the system which also describes the coordinate axis used by the stationary external observer. Thus, the basis vectors $\sigma^{a b}$ are represented by a 4 by 4 matrix of equation 2.24 . The expression of displacement $\Delta x_{\amalg}^{\prime}$ in the new coordinate basis is the product of matrix $\sigma^{a b}$ and displacement $\Delta x_{\longleftarrow}^{\prime}$ as shown below.

$$
\sigma^{a \Perp} \Delta x_{\longleftarrow}^{\prime}=\sigma^{a Ц}\left(t v_{s}\right)\left[1-\frac{2 G\left(N_{a} m_{p}\right)}{r^{\prime}(.99) c^{2}}\right]^{-1}
$$

Where column indices $b$ of matrix $\sigma^{a b}$ is set equal to index ц $(b=ц)$, and where indices ц takes on values of $\{0,1,2,3\}$ of Minkowski space $M^{4}$ (Penrose, 2004). As stated in section 1 , the motion of the system is defined on a geodesic, this fact requires that the displacement $\sigma^{a \Perp} \Delta x_{\amalg}^{\prime}$ as measured by an external stationary observer lie on the tangent plane defined on a differential space-time manifold (as dictated by General relativity) (Penrose, 2004). Moreover, in defining a metric in terms of general relativity or Einstein's field equations, the displacement $\sigma^{a \Perp} \Delta x_{\amalg}^{\prime}$ must be associated with the curved surface of a space-time manifold. The field function $\phi\left(x_{\amalg}^{\prime}(t)\right)$ is a smooth and continuous function of the Minkowski coordinates of $x_{\amalg}^{\prime}=x_{\amalg}^{\prime}(t),\left(x_{0}^{\prime}, x_{1}^{\prime}, x_{2}^{\prime}, x_{3}^{\prime}\right)$ describing a differential space-time manifold (Penrose, 2004), where $x_{\amalg}^{\prime}$ is re-expressed such that $x_{\amalg}^{\prime}=x_{\amalg}^{\prime}(t)=$ $\sigma^{a \Perp} t v_{s}$. The displacement $\sigma^{a \Perp} \Delta x_{Ц}^{\prime}$ is stated to be a displacement on the tangent plane of the differential manifold of field function $\phi\left(x_{\amalg}^{\prime}(t)\right)$ (Penrose, 2004), thus the displacement $\sigma^{a \amalg} \Delta x_{\amalg}^{\prime}$ is expressed as a tangent vector such that:

$$
\frac{\partial \phi\left(x_{\mathrm{L}}^{\prime}(t)\right)}{\partial x_{\mathrm{U}}^{\prime}}=\sigma^{a \Perp} \Delta x_{\mathrm{L}}^{\prime}=\left(\sigma^{a \Perp} t v_{S}\right)\left[1-\frac{2 G\left(N_{a} m_{p}\right)}{r^{\prime}(.99) c^{2}}\right]^{-1}
$$

A very important mathematical clarification to equation 2.26 is that the tangent vector is the partial derivative $\partial \phi\left(x_{\amalg}^{\prime}\right) / \partial x_{\amalg}^{\prime}$ which has a value at Minkowski coordinate $x_{\amalg}^{\prime}$ which takes on a value $\sigma^{a \Perp} t v_{s}$ for the three spatial coordinates $\left(x_{1}^{\prime}, x_{2}^{\prime}\right.$, and $x_{3}^{\prime}$ ). Therefore, in reference to the partial derivative $\partial \phi\left(x_{\amalg}^{\prime}\right) / \partial x_{\amalg}^{\prime}$ (or tangent vector), there is no need for the use of the chain rule $\left(f^{\prime}(x(t))\left(x^{\prime}(t)\right)\right)$ expressing the variation in $x_{\mathrm{u}}^{\prime}$ which vary in respect to parameter time $t$ (velocity $v_{s}$ is a constant). The time coordinate $x_{0}^{\prime}$ is the exception as is shown in equation 2.29 .

In giving an algebraic description of the displacement on the tangent plane, consider smooth functions $\phi\left(x_{\amalg}^{\prime}(t)\right)$ and $f\left(x_{\mathrm{L}}^{\prime}(t)\right)$ at Minkowski coordinates $x_{\mathrm{u}}^{\prime}(t)$ which describe a curved surface of a smooth space-time manifold. The tangent vectors to field functions $\phi\left(x_{\mathrm{U}}^{\prime}(t)\right)$ and $f\left(x_{\mathrm{L}}^{\prime}(t)\right)$ are denoted $T\left[\phi\left(x_{\mathrm{L}}^{\prime}(t)\right)\right]$ and $T\left[f\left(x_{\mathrm{L}}^{\prime}(t)\right)\right]$. The tangent vector function $T\left[\phi\left(x_{\mathrm{L}}^{\prime}(t)\right)\right]$ relates to tangent vector function $T\left[f\left(x_{\mathrm{\amalg}}^{\prime}(t)\right)\right]$ and compression factor $a\left(r_{s}\right)$ such that:

$$
T\left[\phi\left(x_{\amalg}^{\prime}(t)\right)\right]=\left(\left(a\left(r_{s}\right)\right)^{-1}\right) T\left[f\left(x_{\amalg}^{\prime}(t)\right)\right]
$$


Equation 2.27 above conveys the form of equation 2.26. This implies that tangent vector function $T\left[f\left(x_{\amalg}^{\prime}(t)\right)\right]$ has a value such that $T\left[f\left(x_{\amalg}^{\prime}(t)\right)\right]=a\left(r_{s}\right) T\left[\phi\left(x_{\amalg}^{\prime}(t)\right)\right]$. The tangent vector function of $T\left[f\left(x_{\mathrm{U}}^{\prime}(t)\right)\right]$ represents variations on the space-time curve of field function $f\left(x_{\mathrm{L}}^{\prime}(t)\right)$ compressed by compression factor $a\left(r_{s}\right)$. However and as previously stated, the motion of the system traveling at velocity $v_{s}$ (and therefore displacement $\left.\sigma^{a \Perp} \Delta x_{\amalg}^{\prime}\right)$ is measured by the amount of compressed spatial distance crossed as compared to an equal amount of uncompressed distance crossed as if it were in flat or linear space (as measured by a stationary observer outside of the generated gravitational field). Hence showing the use of the inverse compression factor $\left(a\left(r_{s}\right)\right)^{-1}$ as measured on the tangent plane.

At this juncture, the displacement $\sigma^{a \Perp} \Delta x_{ц}^{\prime}$ has been sufficiently related to the tangent plane embedded on the surface of a differential manifold of field function $\left(x_{\mathrm{\Psi}}^{\prime}(t)\right)$. Tangent vector $\partial \phi\left(x_{\mathrm{U}}^{\prime}(t)\right) / \partial x_{\mathrm{\bigsqcup}}^{\prime}$ is an element of $R^{4}$ and represents an instantaneous rate of change in time and distance on the tangent plane (Wald, 1984). The scalar quantity of the differential element of displacement in time $t$ is denoted $d t\left(d t \in R^{1}\right)$, this is multiplied to the tangent vector such that (Wald, 1984) :

$$
\frac{\partial \phi\left(x_{\Perp}^{\prime}(t)\right)}{\partial x_{\Perp}^{\prime}} d t=\left(\sigma^{a \Perp}\left(t v_{s}\right)\left[1-\frac{2 G\left(N_{a} m_{p}\right)}{r^{\prime}(.99) c^{2}}\right]^{-1}\right) d t
$$

Therefore, equation 2.28 is a vector quantity in $M^{4}$ giving the distance on the tangent plane in respect to time $t$. It is pertinent to recognize that the tangent vector component at the coordinate $x_{0}^{\prime}$ (or $x_{0}^{\prime}(t)$ ) in $M^{4}$ (the 4-space Minkowski coordinates) takes on a value such that (Penrose, 2004):

$$
\frac{\partial \phi\left(x_{0}^{\prime}(t)\right)}{\partial x_{0}^{\prime}} d t=\frac{\partial \phi\left(x_{0}^{\prime}(t)\right)}{\partial x_{0}^{\prime}} \frac{\partial x_{0}^{\prime}}{\partial t} d t=\frac{\partial(c i t)}{\partial t} d t=c i d t \equiv \sigma^{a 0} \Delta x_{0}^{\prime} d t
$$

Where the chain rule is incorporated into equation 2.29, this implies that the basis vector at $\sigma^{00}$ has a value of one $\left(\sigma^{00}=1\right)$ for component $x_{0}^{\prime}(t)$. Complex time component $\left(x_{0}^{\prime}=x_{0}^{\prime}(t)\right)$ is the product of the speed of light $c$ and time $t\left(x_{0}^{\prime}=c i t\right)$ in accordance to the Minkowski coordinates (Penrose, 2004) and the speed of light is set to unity $(c=1)$. Equation 2.29 is the tangent vector to the field $\phi\left(x_{\mathrm{L}}^{\prime}(t)\right)$ (Penrose, 2004) at component $x_{0}^{\prime}$ $\left(\phi\left(x_{0}^{\prime}(t)\right)\right)$. The tangent vectors for field function $\phi\left(x_{\amalg}^{\prime}(t)\right)$ at components $x_{1}^{\prime}(t)-x_{3}^{\prime}(t)$ (or the space-like components) are of the form of displacement $\sigma^{a \Perp} \Delta x_{\longleftarrow}^{\prime}$ of equation 2.28. Observe the symmetric tangent vector components below.

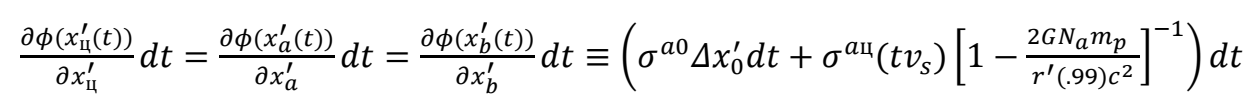

Where the symmetric terms of equation 2.30 are vector valued quantities in $M^{4}$, the differential volume element $\partial x_{\amalg}^{\prime}$ in $M^{4}\left(\partial x_{\amalg}^{\prime} \in M^{4}\right)$ in correspondence to the variations in $x_{\amalg}^{\prime}=x_{\amalg}^{\prime}(t)$ is multiplied to the tangent vector giving the linear combination of:

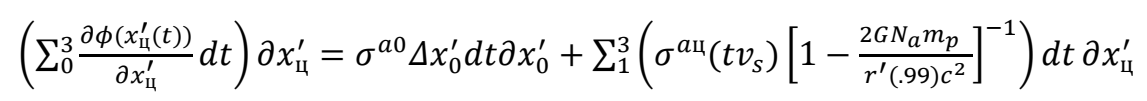

The linear combination of equation 2.31 is a scalar quantity. The symmetric terms can then be stated as superposition values such that:

$$
\sum_{0}^{3} \frac{\partial \phi\left(x_{\amalg}^{\prime}(t)\right)}{\partial x_{\amalg}^{\prime}} d t \partial x_{\amalg}^{\prime}=\sum_{0}^{3} \frac{\partial \phi\left(x_{a}^{\prime}(t)\right)}{\partial x_{a}^{\prime}} d t \partial x_{a}^{\prime}=\sum_{0}^{3} \frac{\partial \phi\left(x_{b}^{\prime}(t)\right)}{\partial x_{b}^{\prime}} d t \partial x_{b}^{\prime}
$$

The Kronecker delta $\delta^{a b}$ is expressed such that (Wald, 1984):

$$
\delta^{a b}=\left\{\begin{array}{l}
a=b, \delta^{a b}=1 \\
a \neq b, \delta^{a b}=0
\end{array}\right.
$$

Where the product of symmetric superposition terms is expressed such that:

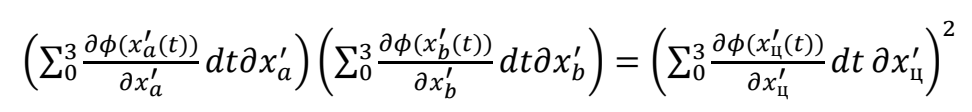

Applying the Kronecker delta to the product of 2.34 above gives (Wald, 1984):

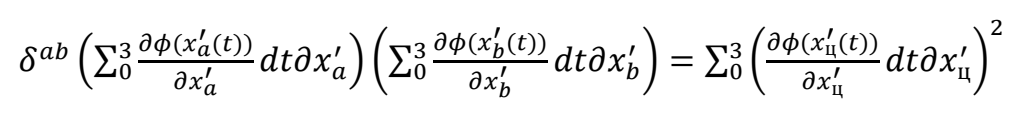


One obtains the Pythagorean relation on the tangent plane to the space-time manifold which is a metric denoted $d s^{2}$. Metric $d s^{2}$ is expressed such that (Wald, 1984):

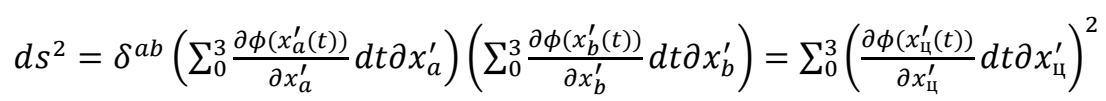

The component of metric $d s^{2}$ at the time coordinate $x_{0}^{\prime}$ has a value of $-d t^{2} \partial\left(x_{\amalg}^{\prime}\right)^{2}\left(x_{0}^{\prime}=i t\right)$ in accordance to equation 2.29, thus metric $d s^{2}$ can be expressed such that:

$$
d s^{2}=-d t^{2} \partial\left(x_{0}^{\prime}\right)^{2}+\sum_{1}^{3}\left(\frac{\partial \phi\left(x_{\amalg}^{\prime}(t)\right)}{\partial x_{\Perp}^{\prime}} d t \partial x_{\longleftarrow}^{\prime}\right)^{2}
$$

Recall that the basis vector at $\sigma^{00}$ has a value of one $\left(\sigma^{00}=1\right)$ and the speed of light is set to unity $(c=1)$. Substituting the value of equation 2.25 into equation 2.37, one obtains (Wald, 1984):

$$
d s^{2}=-d t^{2} \partial\left(x_{0}^{\prime}\right)^{2}+\sum_{1}^{3}\left(\left[\sigma^{a \Perp}\left(t v_{s}\right)\left[1-\frac{2 G N_{a} m_{p}}{r^{\prime}(.99) c^{2}}\right]^{-1}\right]\right)^{2} d t^{2} \partial\left(x_{\longleftarrow}^{\prime}\right)^{2}
$$

The metric of equation 2.38 shown above is the "Velocity Based Gravitation by Accelerated Particles metric" (VBGAP) due to the fact that the displacement through space-time is measured at the system velocity $v_{s}$ (where velocity $v_{s}$ is less than the speed of light $v_{s}<c$ ) and the compression or contraction of space-time $a\left(r_{s}\right)$ caused by the gravitational fields generated by the Particle accelerators. Equation 2.37 can be stated as the alternative to the Alcubierre metric. A priori is that the VBGAP metric can be related to the length of a curve over the surface of field function $\phi\left(x_{\mathrm{L}}^{\prime}(t)\right)$ for initial and final time values $t_{i}$ and $t_{f}$ as shown below.

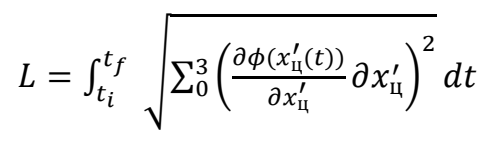

The simplest form of the smooth and differentiable field function $\phi\left(x_{\mathrm{L}}^{\prime}(t)\right)$ describing the gravitational space-time curve surface generated by the particle accelerator is expressed such that:

$$
\phi\left(x_{\amalg}^{\prime}(t)\right)=x_{0}+\frac{1}{2}\left[\left(a\left(r_{s}\right)\right)^{-1}\left[\left(x_{1}\right)^{2}+\left(x_{2}\right)^{2}+\left(x_{3}\right)^{2}\right]\right]
$$

Where the coordinate $x_{\amalg}^{\prime}$ is of the form $\sigma^{a \Perp} t v_{s}\left(x_{\amalg}^{\prime}=x_{\amalg}^{\prime}(t)=\sigma^{a \Perp} t v_{s}\right)$, function $\phi\left(x_{\amalg}^{\prime}(t)\right)$ Lastly, the spatial velocity as measured by a stationary observer outside of the volume of curved space corresponding to the gravitational field generated by the particle accelerators is given such that:

$$
v_{r e l}=\frac{x_{\amalg}^{\prime}\left(a\left(r_{S}\right)\right)^{-1}}{t}=v_{S}\left[1-\frac{2 G N_{a} m_{p}}{r^{\prime}(.99) c^{2}}\right]^{-1}
$$

Where velocity $v_{r e l}$ is greater than or equal to system velocity $v_{s}\left(v_{r e l} \geq v_{s}\right)$. An important note is that gravitational time dilation will hinder the calculations of equations 2.38 and 2.41 , the solution to this issue is the arrangement of two gravitational fields of equal magnitude producing an area of flat space-time between the two fields as will be shown in section 4.

\section{The Incorporation of the VBGAP Metric to Einstein's Field Equations}

Both the Alcubierre metric and the VBGAP metric describe warped space-time in terms of General relativity, for instance, the hypothetical vehicles described in both theoretical descriptions ride geodesic world lines on a space-time manifold. Thus, both theoretical descriptions incorporate aspects of Einstein's field equations which require that both theoretical descriptions be compatible to the field equations of General relativity. The most prudent approach to showing that the VBGAP metric is compatible to General relativity and hence Einstein's field equations is to derive the entire equation beginning with and based on the VBGAP metric. Hence, we now begin the heuristic derivation of Einstein's field equations with the VBGAP metric shown below.

$$
d s^{2}=-d t^{2} \partial\left(x_{0}^{\prime}\right)^{2}+\sum_{1}^{3}\left(\left[\sigma^{a \Perp}\left(t v_{s}\right)\left[1-\frac{2 G N_{a} m_{p}}{r^{\prime}(.99) c^{2}}\right]^{-1}\right]\right)^{2} d t^{2} \partial\left(x_{\longleftarrow}^{\prime}\right)^{2}
$$

For mathematical convenience, the components of equation 3.0 are marshaled into vector valued symmetric terms which are the same as equation 2.30 in $M^{4}$ (or Minkowski 4-space $M^{4}$ ) and are equivalent such that (Wald, 1984): 


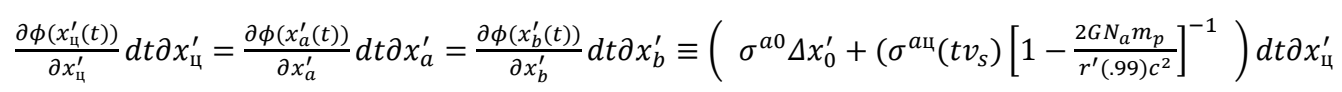

The VBGAP metric $d s^{2}$ can alternatively be re-expressed as the product (or dot product) of vector valued symmetric differential terms such that:

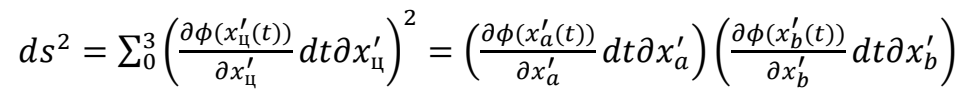

This can be re-arranged such that:

$$
d s^{2}=\left(\frac{\partial \phi\left(x_{a}^{\prime}(t)\right)}{\partial x_{a}^{\prime}} \frac{\partial \phi\left(x_{b}^{\prime}(t)\right)}{\partial x_{b}^{\prime}}\right)\left(d t \partial x_{a}^{\prime}\right)\left(d t \partial x_{b}^{\prime}\right)
$$

The VBGAP metric is set equal to the metric tensor $g_{a b}\left(d s^{2}=g_{a b}\right)$, this can be expressed such that (Wald, 1984):

$$
g_{a b}=\left(\frac{\partial \phi\left(x_{a}^{\prime}(t)\right)}{\partial x_{a}^{\prime}} \frac{\partial \phi\left(x_{b}^{\prime}(t)\right)}{\partial x_{b}^{\prime}}\right)\left(d t \partial x_{a}^{\prime}\right)\left(d t \partial x_{b}^{\prime}\right)
$$

We must acknowledge the fact that when a coordinate $x_{c}^{\prime}$ is parameterized by time $t$, many authors denote the metric tensor as $g_{a b} \frac{\partial x_{a}}{\partial t} \frac{\partial x_{b}}{\partial t}$, however to avoid a cluttered appearance in the mathematical exposition of the content, we simply use $g_{a b}$ (Wald, 1984). The geodesic rule is given such that $\nabla_{c} g_{a b}=0$ (Wald, 1984). Therefore, we look to obtain the straightest possible curve on the field function $\phi\left(x_{\mathrm{L}}^{\prime}(t)\right)$ of the space-time manifold, the derivative $\nabla_{c}$ is given such that (Wald, 1984):

$$
\nabla_{c}=\frac{\partial}{\partial x_{c}^{\prime}}=\frac{\partial}{\partial x_{c}^{\prime}} \frac{\partial x_{c}^{\prime}}{\partial t} \equiv \frac{\partial}{\partial x_{c}^{\prime}(t)} \frac{\partial x_{c}^{\prime}(t)}{\partial t}
$$

The chain rule must be applied due to the fact that the value of $x_{c}^{\prime}$ is parameterized by time $t\left(x_{c}^{\prime}=x_{c}^{\prime}(t)=\right.$ $\left.x_{\amalg}^{\prime} \equiv \sigma^{a \Perp} t v_{s}\right)$. The derivative $\nabla_{c}$ is applied to the value of equation 3.04, resultantly one obtains equation 3.06 below (Wald, 1984).

$$
\nabla_{c} g_{a b}=\nabla_{c}\left(\left(\frac{\partial \phi\left(x_{a}^{\prime}(t)\right)}{\partial x_{a}^{\prime}} \frac{\partial \phi\left(x_{b}^{\prime}(t)\right)}{\partial x_{b}^{\prime}}\right)\left(d t \partial x_{a}^{\prime}\right)\left(d t \partial x_{b}^{\prime}\right)\right)
$$

The partial derivative $\nabla_{c}$ acting on each symmetric vector valued component of expression 3.02 has individual chain rule expressions in accordance with equation 3.05 of the form:

$$
\nabla_{c}\left(\frac{\partial \phi\left(x_{c}^{\prime}(t)\right)}{\partial x_{c}^{\prime}} d t \partial x_{c}^{\prime}\right)=\frac{\partial}{\partial x_{c}^{\prime}}\left(\left(\sigma^{a 0} \Delta x_{0}^{\prime}+\left(\sigma^{a c} x_{c}^{\prime}(t)\right)\left[1-\frac{2 G N_{a} m_{p}}{r^{\prime}(.99) c^{2}}\right]^{-1}\right) d t \partial x_{c}^{\prime}\right) \frac{\partial x_{c}^{\prime}}{\partial t}
$$

Where $x_{c}^{\prime}=x_{c}^{\prime}(t)=\sigma^{a c} t v_{s}$, the parts of the chain rule expression $\left(\partial / \partial x_{c}^{\prime}\right.$ and $\left.\partial x_{c}^{\prime} / \partial t\right)$ of the derivative $\nabla_{c}$ acting on equation 3.07 have values of:

$$
\begin{gathered}
\frac{\partial}{\partial x_{c}^{\prime}}\left(\frac{\partial \phi\left(x_{c}^{\prime}(t)\right)}{\partial x_{c}^{\prime}} d t \partial x_{c}^{\prime}\right)=\left(\left(\left[1-\frac{2 G N_{a} m_{p}}{r^{\prime}(.99) c^{2}}\right]^{-1}\right) d t \partial x_{c}^{\prime}\right) \\
\frac{\partial x_{c}^{\prime}}{\partial t}=\frac{\partial\left(\sigma^{a c} t v_{S}\right)}{\partial t}=\left(0, \sigma^{a 1} v_{s}, \sigma^{a 2} v_{s}, \sigma^{a 3} v_{s}\right)
\end{gathered}
$$

Applying the geodesic condition to equation 3.06, equation 3.06 is set equal to zero giving:

$$
\nabla_{c} g_{a b}=\frac{\partial}{\partial x_{c}^{\prime}}\left(\frac{\partial \phi\left(x_{a}^{\prime}(t)\right)}{\partial x_{a}^{\prime}} \frac{\partial \phi\left(x_{b}^{\prime}(t)\right)}{\partial x_{b}^{\prime}}\right) d t \partial x_{a}^{\prime} d t \partial x_{b}^{\prime} \equiv 0
$$

Thus applying the product rule or leibnitz rule to equation 3.10 above gives (Wald, 1984):

$$
\nabla_{c} g_{a b}=\frac{\partial}{\partial x_{a}^{\prime}}\left(\frac{\partial \phi\left(x_{a}^{\prime}(t)\right)}{\partial x_{c}^{\prime}}\right) \frac{\partial \phi\left(x_{b}^{\prime}(t)\right)}{\partial x_{b}^{\prime}} d t \partial x_{a}^{\prime} d t \partial x_{b}^{\prime}+\frac{\partial}{\partial x_{b}^{\prime}} \frac{\partial \phi\left(x_{a}^{\prime}(t)\right)}{\partial x_{a}^{\prime}}\left(\frac{\partial \phi\left(x_{b}^{\prime}(t)\right)}{\partial x_{c}^{\prime}}\right) d t \partial x_{a}^{\prime} d t \partial x_{b}^{\prime} \equiv 0
$$

This (equation 3.11) is equivalently expressed in short hand notation such that (Wald, 1984):

$$
\nabla_{c} g_{a b}=\nabla_{a} g_{c b}+\nabla_{b} g_{a c} \equiv 0
$$

Keep in mind that the terms are all symmetric $\left(\nabla_{a} g_{c b}=\nabla_{b} g_{a c}=\nabla_{c} g_{a b}\right)$ (Wald, 1984). Equation 3.11 can be algebraically arranged such that (Wald, 1984): 


$$
\nabla_{a} g_{c b}+\nabla_{b} g_{a c}-\nabla_{c} g_{a b} \equiv 0
$$

Where $C_{c a b}=\nabla_{c} g_{a b}$, this implies that the terms of $C_{c a b}$ are symmetric $\left(C_{c a b}=C_{b a c}=C_{a c b}\right)$ (Wald, 1984). An equivalence to equation 3.13 is given such that (Wald, 1984):

$$
C_{a c b}+C_{b a c}-C_{c a b}=\nabla_{a} g_{c b}+\nabla_{b} g_{a c}-\nabla_{c} g_{a b} \equiv 0
$$

Observe the term $C_{b a c}$ which has a value $C_{b a c}=\nabla_{b} g_{a c}$ in equation 3.14 above (Wald, 1984). Applying the product rule or liebnitz rule to term $C_{b a c}$, the term expand such that (Wald, 1984):

$$
C_{b a c}=\nabla_{a} g_{b c}+\nabla_{c} g_{a b} \equiv C_{a b c}+C_{c a b}
$$

This can be expressed such that (Wald, 1984):

$$
C_{b a c}=C_{a b c}+C_{c a b}
$$

Substituting this into the original equation (3.14) gives (Wald, 1984):

$$
C_{a c b}+\left[C_{a b c}+C_{c a b}\right]-C_{c a b}=\nabla_{a} g_{c b}+\nabla_{b} g_{a c}-\nabla_{c} g_{a b} \equiv 0
$$

The symmetric property is again applied $\left(C_{c a b}=C_{b a c}=C_{a c b}\right)$, therefore the left side of equation 3.17 above can be reduced such that (Wald, 1984):

This implies that (Wald, 1984):

$$
2 C_{a c b}=\nabla_{a} g_{c b}+\nabla_{b} g_{a c}-\nabla_{c} g_{a b} \equiv 0
$$

$$
C_{a c b}=\frac{1}{2}\left\{\nabla_{a} g_{c b}+\nabla_{b} g_{a c}-\nabla_{c} g_{a b}\right\}
$$

The inverse tensor $g^{a b}$ (such that $g^{a b} \bullet g_{a b}=I$, where $I$ is the 4 by 4 identity matrix) is applied to the equation above such that (Wald, 1984):

$$
g^{a b} C_{a c b}=\frac{g^{a b}}{2}\left\{\nabla_{a} g_{c b}+\nabla_{b} g_{a c}-\nabla_{c} g_{a b}\right\}
$$

The values of the metric tensors $g_{a b}$ are symmetric $\left(g_{a b}=g_{c b}=g_{c a}=\cdots_{\text {... }}\right)$ permiting the distribution of the inverse tensor $g^{a b}$ over equation 3.20 (Wald, 1984). Implementing the distributive property, one obtains an expression in terms of partial derivatives, where $\nabla_{x}$ is the derivative of the straightest possible world line on the curved surface of the space-time manifold (Wald, 1984).

$$
\nabla_{x}=g^{a b} C_{a c b}=\frac{1}{2}\left\{\nabla_{a}+\nabla_{b}-\nabla_{c}\right\}
$$

Setting this product equal to the Christoffel symbol $\Gamma_{c b}^{a}$ gives (Wald, 1984) :

$$
\Gamma_{c b}^{a}=g^{a b} C_{a c b} \equiv \nabla_{x}
$$

Therefore this can be expressed such that (Wald, 1984):

$$
\Gamma_{c b}^{a}=\frac{g^{a b}}{2}\left\{\nabla_{a} g_{c b}+\nabla_{b} g_{a c}-\nabla_{c} g_{a b}\right\}
$$

Where $\nabla_{a}=\nabla_{b}=\nabla_{c}=\nabla_{d}$ are symmetric partial derivatives, subtracting the value of $2 \Gamma_{c b}^{a}$ from the derivative of $\nabla_{c}$ permits the expression to equal zero as shown below (Wald, 1984).

$$
\nabla_{c}=\nabla_{c}-2 \Gamma_{c b}^{a}=\nabla_{c}-2 \nabla_{x} \equiv 0
$$

Re-applying the metric tensor $g_{a b}$ to equation 3.24, one obtains (Wald, 1984):

Hence one obtains:

$$
\nabla_{c} g_{a b}=\nabla_{c} g_{a b}-2 g_{a b} \Gamma_{c b}^{a}=\nabla_{c} g_{a b}-g_{a b} 2 \nabla_{x} \equiv 0
$$

$$
\nabla_{c} g_{a b}=\nabla_{c} g_{a b}-2 g_{a b} \Gamma_{c b}^{a} \equiv 0
$$

Thus we have derived the geodesic equation (Wald, 1984). To verify the values of the VBGAP metric to the geodesic equation, simply substitute the VBGAP metric values of partial derivatives of equations 3.08 and 3.09 into equation 3.26. The values of equations 3.08 and 3.09 will satisfy equation 3.26 above. The values of partial derivatives $\nabla_{s}$ and $\nabla_{t}$ are of the form of the geodesic equation as shown below (Wald, 1984).

$$
\nabla_{s}=\nabla_{s}-2 \Gamma_{c b}^{a} \equiv 0 \quad \nabla_{t}=\nabla_{t}-2 \Gamma_{c b}^{a} \equiv 0
$$

As stated by Wald (Wald, 1984), the commutator for computing curvature is composed of derivatives $\nabla_{s}$ and $\nabla_{t}$ as shown below. 


$$
\left[\nabla_{s}, \nabla_{t}\right]=\nabla_{s} \nabla_{t}-\nabla_{t} \nabla_{s} \quad \equiv\left(\nabla_{s}-2 \Gamma_{c b}^{a}\right)\left(\nabla_{t}-2 \Gamma_{c b}^{a}\right)-\left(\nabla_{t}-2 \Gamma_{c b}^{a}\right)\left(\nabla_{s}-2 \Gamma_{c b}^{a}\right)
$$

Therefore as stated by Wald (Wald, 1984), the Ricci tensor $R_{a b}$ is the product of the commutator for computing curvature $\left[\nabla_{s}, \nabla_{t}\right]$ and the metric tensor $g_{a b}$ given such that:

$$
R_{a b}=\left[\nabla_{s}, \nabla_{t}\right] g_{a b}
$$

Thus the Einstein tensor $G_{a b}$ is expressed such that (Wald, 1984):

$$
G_{a b}=R_{a b}-\frac{g_{a b} R}{2} \equiv 0
$$

Where $R$ is the scalar curvature computed by the coordinate component method or the orthonormal basis (Tetrad) method (Wald, 1984). Equation 3.31 can alternatively be expressed such that:

$$
G_{a b}=\left[\nabla_{s}, \nabla_{t}\right] g_{a b}-\frac{g_{a b} R}{2}
$$

Substituting the value of VBGAP metric (where $d s^{2}=g_{a b}$ ) into the Einstein tensor $G_{a b}$ or equation 3.31 gives the expression (Wald, 1984):

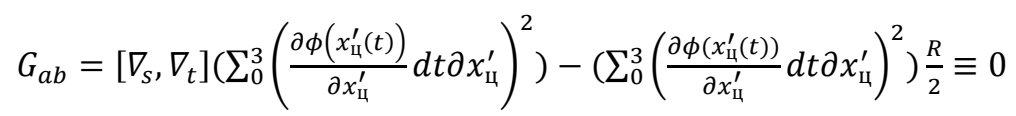

Conclusively, the Einstein tensor can be expressed in terms of the values of the VBGAP metric such that:

$$
G_{a b}=\left(\left[\nabla_{s}, \nabla_{t}\right]-\frac{R}{2}\right)\left(-d t^{2} \partial\left(x_{0}^{\prime}\right)^{2}+\sum_{1}^{3}\left(\left[\sigma^{a \Perp}\left(x_{\text {Ц }}^{\prime}\right)\left[1-\frac{2 G N_{a} m_{p}}{r^{\prime}(.99) c^{2}}\right]^{-1}\right]\right)^{2} d t^{2} \partial\left(x_{\longleftrightarrow}^{\prime}\right)^{2}\right) \equiv 0
$$

Setting the Einstein tensor $G_{a b}$ equal to the stress energy tensor $8 \pi T_{a b}\left(G_{a b}=8 \pi T_{a b}\right)$ gives (Wald, 1984):

$$
8 \pi T_{a b}=\left(\left[\nabla_{s}, \nabla_{t}\right]-\frac{R}{2}\right)\left(-d t^{2} \partial\left(x_{0}^{\prime}\right)^{2}+\sum_{1}^{3}\left(\left[\sigma^{a \Perp}\left(t v_{s}\right)\left[1-\frac{2 G N_{a} m_{p}}{r^{\prime}(.99) c^{2}}\right]^{-1}\right]\right)^{2} d t^{2} \partial\left(x_{\longleftrightarrow}^{\prime}\right)^{2}\right)
$$

The formulation of the stress-energy tensor $8 \pi T_{a b}$ in terms of the VBGAP metric is left as an exercise. Equations 3.31, 3.32, and 3.33 accomplish the goal of showing that the VBGAP metric has been formulated in accordance to Einstein's field equations

\section{The Solution to the Gravitational Time Dilation Problem with Generated Gravitation Fields}

As dictated and proven by general relativity, the gravitational fields generated by the particle accelerators will cause time to "slowdown" as compared to flat space located beyond the effects of the gravitational field. Resultantly, as the system moves spatially with a velocity $v_{s}$ over a proper time $t$, time will be dilated by the gravitational field generated by the particle accelerators. The system will be traveling at a slower temporal or time-like rate as compared to linear or flat space which will diminish the system's space-like motion which is spatial displacement $x_{\amalg}^{\prime}\left(x_{\amalg}^{\prime}=\sigma^{a c} t v_{S}\right)$. Therefore, despite the system's compression of space-time in the direction of displacement $x_{\amalg}^{\prime}$, an object traveling in the same direction and velocity $\left(v_{s}\right)$ in the same inertial frame but outside of (or beyond) the effects of the generated gravitational fields in flat or linear space will be traveling relatively faster. Section 4 gives the solution to the gravitational time dilation issue with presenting the notion of an equilibrium point between two gravitational fields produced by two particles accelerators that will compensate for the time slippage inherently caused by gravity.

Consider two gravitational force fields $F_{g 1}^{\prime}$ and $F_{g 2}^{\prime}$ (in the form of equation 2.10) produced by two particle accelerators. The acceleration numbers $N_{a 1}$ and $N_{a 2}$ correspond to gravitational forces $F_{g 1}^{\prime}$ and $F_{g 2}^{\prime}$. Gravitational forces $F_{g 1}^{\prime}$ and $F_{g 2}^{\prime}$ at distance values $r_{1}$ and $r_{2}$ take on values and conditions such that:

$$
\begin{array}{lll}
F_{g 1}^{\prime}=\frac{G N_{a 1} m_{p 1} m_{0}}{\left.\left(r_{1}\right)^{2} .99\right)}, & N_{a 1} \in R, & N_{a 1} \geq 1 \\
F_{g 2}^{\prime}=\frac{G N_{a 2} m_{p 2} m_{0}}{\left(r_{2}\right)^{2}(.99)}, & N_{a 2} \in R, & N_{a 2} \geq 1
\end{array}
$$

Where $m_{0}$ is the mass of a test particle, and masses $m_{p 1}$ and $m_{p 2}$ (where $m_{p 1}=m_{p 2}$ ) are the mass values of the accelerated particles in each particle accelerator, the Gravitational forces $F_{g 1}^{\prime}$ and $F_{g 2}^{\prime}$ are equal in magnitude $\left(F_{g 1}^{\prime}=F_{g_{2}}^{\prime}\right)$, this equivalence is expressed such that:

$$
\frac{G N_{a 1} m_{p 1} m_{0}}{\left(r_{1}\right)^{2}(.99)}=\frac{G N_{a 2} m_{p 2} m_{0}}{\left(r_{2}\right)^{2}(.99)}
$$


The gravitational forces $F_{g 1}^{\prime}$ and $F_{g 2}^{\prime}$ have an equilibrium point between them at spatial coordinated $x_{0}$, where the forces are equal. Mass value $m_{0}$ is located at equilibrium coordinate $x_{0}$. Equilibrium coordinate $x_{0}$ is a distance $r_{1}$ from gravitational force $F_{g 1}^{\prime}$ (or the particle accelerator). Hence, equilibrium coordinate $x_{0}$ is a distance $r_{2}$ from gravitational force $F_{g 2}^{\prime}$ (or the second particle accelerator). Force value $F\left(r_{1}, r_{2}\right)$ is a function of distance values $r_{1}$ and $r_{2}$ and is the difference between force values $F_{g 1}^{\prime}$ and $F_{g 2}^{\prime}$. Thus the combined force $F\left(r_{1}, r_{2}\right)$ is the total value of gravitational force acting or pulling on mass $m_{0}$ at the equilibrium coordinate $x_{0}$ located between both particle accelerators which implies that force value $F\left(r_{1}, r_{2}\right)$ is equal to zero at equilibrium coordinate $x_{0}$. Force value $F\left(r_{1}, r_{2}\right)$ is then expressed such that:

$$
F\left(r_{1}, r_{2}\right)=F_{g 1}^{\prime}-F_{g 2}^{\prime}=0
$$

This can be expressed such that:

$$
F\left(r_{1}, r_{2}\right)=\frac{G N_{a 1} m_{p} m_{0}}{\left(r_{1}\right)^{2}(.99)}-\frac{G N_{a 2} m_{p} m_{0}}{\left(r_{2}\right)^{2}(.99)}=0
$$

Gravitational potential energy $U_{g}^{\prime}$ is the integral of gravitational force in respect to radius $r$ such that (Young \& Freedman, 2004):

$$
U_{g}^{\prime}=\int F_{g}^{\prime} d r
$$

Therefore, potential energy $U_{g}^{\prime}\left(r_{1}, r_{2}\right)$ is the integral of gravitational force $F\left(r_{1}, r_{2}\right)$ in respect to radius $r$ such that:

$$
U_{g}^{\prime}\left(r_{1}, r_{2}\right)=\int F\left(r_{1}, r_{2}\right) d r=\int\left[F_{g 1}^{\prime}-F_{g 2}^{\prime}\right] d r=0
$$

Where $a_{1}$ is the initial point at the first accelerator and equilibrium point $x_{0}$ is the location of mass $m_{0}$ pertaining to distance $r_{1}$. Coordinate $a_{2}$ is the initial point at the second accelerator and equilibrium point $x_{0}$ is the location of mass $m_{0}$ pertaining to distance $r_{2}$. With aforementioned values being the limits of integration, potential energy $U_{g}^{\prime}\left(r_{1}, r_{2}\right)$ is expressed as the difference between two integrals in respect to $r_{1}$ and $r_{2}$ such that:

$$
U_{g}^{\prime}\left(r_{1}, r_{2}\right)=\int_{a_{1}}^{x_{0}}\left(\frac{G\left(N_{a 1} m_{p 1}\right) m_{0}}{\left.\left(r_{1}\right)^{2} .99\right)}\right) d r_{1}-\int_{a_{2}}^{x_{0}}\left(\frac{G\left(N_{a 2} m_{p 2}\right) m_{0}}{\left(r_{2}\right)^{2}(.99)}\right) d r_{2}=0
$$

Potential energy $U_{g}^{\prime}\left(r_{1}, r_{2}\right)$ takes on a value of zero $\left(U_{g}^{\prime}\left(r_{1}, r_{2}\right)=0\right)$. Potential energy $U_{g}^{\prime}\left(r_{1}, r_{2}\right)$ is the value of energy at the equilibrium coordinate $x_{0}$ between the two particle accelerators. A free falling particle of mass $m_{0}$ with an initial velocity of zero located at the equilibrium coordinate $x_{0}$ between gravitational forces $F_{g 1}^{\prime}$ and $F_{g 2}^{\prime}$ would have no work done on it and hence the resultant velocity induced by the two gravitational fields would be zero. Gravitational time dilation is expressed such that (Young \& Freedman, 2004; Bench, 2016):

$$
t_{0}=t \sqrt{1-\frac{\left(v_{e S}\right)^{2}}{c^{2}}}=t \sqrt{1-\frac{1}{c^{2}}\left(\frac{2 G M}{r}\right)}
$$

Time $t_{0}$ is dilated time and time $t$ is the proper time for the purposes of the explanation (Young \& Freedman, 2004). Kinetic energy $k$ is the energy of a particle of mass $m_{0}$ at the velocity $v_{e s}$ which is the escape velocity required for the gravitational fields as shown below (Young \& Freedman, 2004).

$$
k=\frac{m_{0}\left(v_{e s}\right)^{2}}{2}
$$

Kinetic energy $k$ is set equal to potential energy $U_{g}^{\prime}\left(r_{1}, r_{2}\right)\left(k=U_{g}^{\prime}\left(r_{1}, r_{2}\right)\right)$ of equation 4.07 giving (Young \& Freedman, 2004):

$$
\frac{m_{0}\left(v_{e s}\right)^{2}}{2}=U_{g}^{\prime}\left(r_{1}, r_{2}\right)=0
$$

This implies that:

$$
v_{e s}=\sqrt{U_{g}^{\prime}\left(r_{1}, r_{2}\right)}=0
$$

Hence, velocity $v_{e s}$ is equal to zero $\left(v_{e s}=0\right)$ at the equilibrium coordinate $x_{0}$ between gravitational forces $F_{g 1}^{\prime}$ and $F_{g 2}^{\prime}$. Time $t_{0}$ of equation 4.07 has a value at velocity $v_{e s}=0$ such that:

$$
t_{0}=t \sqrt{1-\frac{(0)^{2}}{c^{2}}}=t
$$


Therefore,

$$
t_{0}=t
$$

Conclusively, at the equilibrium coordinate $x_{0}$ between the two gravitational fields $F_{g 1}^{\prime}$ and $F_{g 2}^{\prime}$, the time value is the same (at proper time $t$ ) for an observer in flat or linear space. Every position $x_{\mathrm{L}}$ within the two gravitational fields $F_{g 1}^{\prime}$ and $F_{g 2}^{\prime}$ that is not at equilibrium coordinate $x_{0}\left(x_{0} \neq x_{\longleftarrow}\right)$ will experience time dilation or temporal slippage $\left(t_{0}<t\right)$ produced by the gravitational fields.

\section{The Two Gravity-Source Warp Field}

This section gives the mathematical description of a hypothetical vehicle traveling at a sub- light velocity (velocity $v_{s}$ ) hoisting two particle accelerators that generate two gravitational fields of equal magnitude which produce an area of flat space-time at an equilibrium point (coordinate $x_{0}$ ) in between them as shown in the previous section. Hence an area of flat space-time is produced within the structure of the vehicle at equilibrium coordinate $x_{0}$. As the vehicle transits space-time, the combined gravitational field produced by both particle accelerators compress space-time (and therefore spatial distance) toward the equilibrium coordinate $x_{0}$ in the vehicle's direction of travel. Equilibrium coordinate $x_{0}$ is the center of motion for the hypothetical vehicle. Therefore VBGAP metric is defined in terms of two gravitational fields whose space-time compression is measured from the equilibrium coordinate $x_{0}$. This warping of space-time around the hypothetical vehicle generated by two particle accelerators of equal magnitude is referred to as the two gravity source warp field.

Two gravitational force fields $F_{g 1}^{\prime}$ and $F_{g 2}^{\prime}$ (of equations 4.0 and 4.01 ) generated by two particle accelerators have equal magnitudes $\left(\left|F_{g 1}^{\prime}\right|=\left|F_{g 2}^{\prime}\right|\right)$ and have an equilibrium point $x_{0}$ between them as defined in the section 4. Thus the variable mass equations $m_{1}^{\prime}$ and $m_{2}^{\prime}$ (of equation 2.05 ) corresponding to gravitational force fields $F_{g 1}^{\prime}$ and $F_{g 2}^{\prime}$ are stated such that:

$$
\begin{array}{ll}
m_{1}^{\prime}=\frac{N_{a 1} m_{p 1}}{.99} & N_{a 1}, \in R, \quad N_{a 1} \geq 1 \\
m_{2}^{\prime}=\frac{N_{a 2} m_{p 2}}{.99} & N_{a 2} \in R, \quad N_{a 2} \geq 1
\end{array}
$$

Variations in gravitational force fields $F_{g 1}^{\prime}$ and $F_{g 2}^{\prime}$ correspond to variations in mass values $m_{1}^{\prime}$ and $m_{2}^{\prime}$ for acceleration numbers $N_{a 1}$ and $N_{a 2}$ shown below.

$$
F_{g 1}^{\prime} \rightarrow m_{1}^{\prime} \rightarrow N_{a 1} \quad F_{g 2}^{\prime} \rightarrow m_{2}^{\prime} \rightarrow N_{a 2}
$$

The variable mass values $m_{1}^{\prime}$ and $m_{2}^{\prime}$ are equal $\left(m_{1}^{\prime}=m_{2}^{\prime}\right)$. The vehicle velocity $v_{s}$ and the compression or contraction of space-time are measured from the equilibrium coordinate $x_{0}$ which is also located at the center of mass $r_{c m}$ due to the gravitational fields $F_{g 1}^{\prime}$ and $F_{g 2}^{\prime}$ having equal magnitudes $\left(\left|F_{g 1}^{\prime}\right|=\left|F_{g 2}^{\prime}\right|\right)$. Center of mass $r_{c m}$ between the two gravitational fields is presented such that (Young \& Freedman, 2004):

$$
r_{c m}=\frac{m_{1}^{\prime} r_{1}+m_{2}^{\prime} r_{2}}{m_{1}^{\prime}+m_{2}^{\prime}} \equiv x_{0}
$$

The distances $r_{1}$ and $r_{2}$ (this implies that $r_{1}=r_{2}$ ) are the distances from the equilibrium coordinate $x_{0}$ between the two gravitational fields $F_{g 1}^{\prime}$ and $F_{g 2}^{\prime}$ of equal magnitude. The two gravitational force fields $F_{g 1}^{\prime}$ and $F_{g 2}^{\prime}$ are in close proximity to one another spatially. Therefore the two masses $m_{1}^{\prime}$ and $m_{2}^{\prime}$ combine to gravitationally attract a test particle of mass value $m_{0}$ which is located at a distance $r$ from equilibrium coordinate $x_{0}$ in the direction of vehicle velocity $v_{s}$ which is the vehicles direction of travel. The magnitude of the combined gravitational force of gravitational force fields $F_{g 1}^{\prime}$ and $F_{g 2}^{\prime}$ are measured from the equilibrium coordinate $x_{0}$ (or center of mass $r_{c m}$ ) which correspond to the reduced mass equation. Observe the reduced mass equation (Young \& Freedman, 2004):

$$
\mu=\frac{m_{1} m_{2}}{m_{1}+m_{2}}
$$

To avoid incorrect use of the reduced mass equation, we must point out the fact that the reduced mass $\mu$ in reference to gravitational interactions between masses $m_{1}$ and $m_{2}$ are not reduced. Therefore, in order to describe a gravitational interaction between masses $m_{1}$ and $m_{2}$ (or $m_{1} m_{2}$ ) incorporating reduced mass, the reduced mass must be expressed such that (Nipoti, 2013):

$$
m_{1} m_{2}=\mu\left(m_{1}+m_{2}\right)=\left(m_{1}+m_{2}\right) \frac{m_{1} m_{2}}{m_{1}+m_{2}}
$$


However, the gravitational force described is not the gravitational force between variable masses $m_{1}^{\prime}$ and $m_{2}^{\prime}$. As previously stated, the combined gravitational force corresponding to the two variable masses $m_{1}^{\prime}$ and $m_{2}^{\prime}$ act on a test particle of mass value $m_{0}$ as measured from the equilibrium coordinate $x_{0}$ (or center of mass $\left.r_{c m}\right)$. Hence, the combined gravitational influence of variable masses $m_{1}^{\prime}$ and $m_{2}^{\prime}$ on mass $m_{0}$ as measured from the equilibrium coordinate $x_{0}$ (or center of mass $r_{c m}$ ) can be described using the reduced mass. Keep in mind that test particle mass $m_{0}$ is a distance $r$ from the equilibrium coordinate $x_{0}$ in the direction of travel. Thus, masses $m_{1}$ and $m_{2}$ in the reduced mass equation (equation 5.04) are substituted by the variable mass values $m_{1}^{\prime}$ and $m_{2}^{\prime}\left(m_{1}=m_{1}^{\prime}, \quad m_{2}=m_{2}^{\prime}\right)$ giving:

$$
\mu^{\prime}=\frac{m_{1}^{\prime} m_{2}^{\prime}}{m_{1}^{\prime}+m_{2}^{\prime}}
$$

This can alternatively be expressed such that:

$$
\mu^{\prime}=\frac{\left(N_{a 1} N_{a 2} m_{p 1} m_{p 2}\right)}{(.99)\left(N_{a 1} m_{p 1}+N_{a 2} m_{p 2}\right)}
$$

The combined gravitational field enacted on mass $m_{0}$ by mass values $m_{1}^{\prime}$ and $m_{2}^{\prime}$ at a distance $r$ as measured from the equilibrium coordinate $x_{0}$ (or center of mass $r_{c m}$ ) between gravitational fields $F_{g 1}^{\prime}$ and $F_{g 2}^{\prime}$ of equal magnitude is expressed by substituting reduced mass $\mu^{\prime}$ for variable mass $m^{\prime}\left(m^{\prime} \rightarrow \mu^{\prime}\right)$ into the gravitational force equation of equation 2.09 shown below (Young \& Freedman, 2004).

$$
F_{g}^{\prime}=\frac{G m^{\prime} m_{0}}{r^{2}}=\frac{G \mu^{\prime} m_{0}}{r^{2}}
$$

Thus one obtains:

$$
F_{g}^{\prime}=\frac{G \mu^{\prime} m_{0}}{r^{2}}=\frac{G\left(N_{a 1} N_{a 2} m_{1} m_{2}\right) m_{0}}{r^{2}(.99)\left(N_{a 1} m_{1}+N_{a 2} m_{2}\right)}
$$

The corresponding value of gravitational potential energy $U_{g}^{\prime}$ is given such that (Young \& Freedman, 2004):

$$
U_{g}^{\prime}=\int F_{g}^{\prime} d r=\int\left[\frac{G \mu^{\prime} m_{0}}{r^{2}}\right] d r
$$

Where the distance from the equilibrium coordinate $x_{0}$ (or center of mass $r_{c m}$ )of radius $r$ is set equal to the Schwarzchild radius $r_{s}\left(r=r_{s}\right)$, potential energy $U_{g}^{\prime}$ is expressed such that:

$$
U_{g}^{\prime}=\frac{G\left(N_{a 1} N_{a 2} m_{1} m_{2}\right) m_{0}}{r_{S}(.99)\left(N_{a 1} m_{1}+N_{a 2} m_{2}\right)}
$$

In repeating the process of equations 2.14-2.16, the corresponding value of the Schwarzchild radius $r_{s}$ incorporating two-source gravity fields generated by the particle accelerators as measured from the equilibrium coordinate $x_{0}$ is expressed such that:

$$
r_{s}=\frac{2 G\left(N_{a 1} N_{a 2} m_{p 1} m_{p 2}\right)}{c^{2}(.99)\left(N_{a 1} m_{p 1}+N_{a 2} m_{p 2}\right)}
$$

Recall that time value $t_{a}$ is a constant value of time, hence, distance $r_{a}$ (equation 2.17) is stated such that:

$$
r_{a}=t_{a} v_{s}
$$

Thus radius $r^{\prime}$ is the sum of Schwarzchild radius $r_{s}$ and distance $r_{a}$ (equation 2.18) such that:

This can be expressed as:

$$
r^{\prime}=r_{a}+r_{s} ; \quad r^{\prime}>r_{s}
$$

$$
r^{\prime}=r_{a}+\frac{2 G\left(N_{a 1} N_{a 2} m_{p 1} m_{p 2}\right)}{c^{2}(.99)\left(N_{a 1} m_{p 1}+N_{a 2} m_{p 2}\right)}
$$

Thus the distance $r^{\prime}$ and therefore Schwarzchild radius $r_{s}$ are measured from the center of mass $r_{c m}$ (equilibrium coordinate $x_{0}$ ) between variable masses $m_{1}^{\prime}$ and $m_{2}^{\prime}$ corresponding to gravitational fields $F_{g 1}^{\prime}$ and $F_{g 2}^{\prime}$ generated by the two particle accelerators. The compression factor denoted $a\left(r_{s}\right)$ for a two-source gravitational field has a value and condition as measured from the equilibrium coordinate $x_{0}$ such that:

$$
a\left(r_{s}\right)=\frac{r^{\prime}-r_{s}}{r^{\prime}}=1-\frac{r_{s}}{r^{\prime}}=1-\frac{2 G\left(N_{a 1} N_{a 2} m_{p 1} m_{p 2}\right)}{c^{2} r^{\prime}(.99)\left(N_{a 1} m_{p 1}+N_{a 2} m_{p 2}\right)} ; \quad 1>a\left(r_{s}\right)
$$


Compression factor $a\left(r_{s}\right)$ is the compression of the geometry of space-time due to gravitational force fields $F_{g 1}^{\prime}$ and $F_{g 2}^{\prime}$ produced by the two particle accelerators as measured from the equilibrium coordinate $x_{0}$. Thus substituting the value of the two-source gravitational field compression factor $a\left(r_{s}\right)$ into equation 2.22 shown below gives,

$$
\Delta x_{\amalg}^{\prime}=x_{\amalg}^{\prime}\left(a\left(r_{S}\right)\right)^{-1}=\frac{t v_{S}}{\left(1-\frac{r_{S}}{r^{\prime}}\right)} ; \quad \Delta x_{\amalg}^{\prime}>x_{\amalg}^{\prime}
$$

And thus the displacement in terms of the two gravity source field is such that:

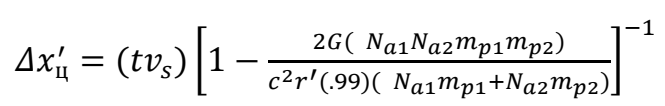

Where the tangent vector component of metric $d s^{2}$ at the time coordinate $x_{0}^{\prime}$ has a differential element of $-d t^{2} \partial\left(x_{0}^{\prime}\right)^{2}$ (equation 2.29), the step by step process of section 2 is again applied accordingly. Applying equation 5.18 to equation 2.28 gives:

$$
\frac{\partial \phi\left(x_{\longleftrightarrow}^{\prime}(t)\right)}{\partial x_{\Perp}^{\prime}} d t=\left(\sigma^{a 0} \Delta x_{0}^{\prime}+\sigma^{a \amalg}\left(t v_{s}\right)\left[1-\frac{2 G\left(N_{a 1} N_{a 2} m_{p 1} m_{p 2}\right)}{c^{2} r^{\prime}(.99)\left(N_{a 1} m_{p 1}+N_{a 2} m_{p 2}\right)}\right]^{-1}\right) d t
$$

Applying equation 5.19 to equation 2.38 gives the VBGAP metric in terms a two gravity source field as measured from the equilibrium coordinate $x_{0}$ which is the vehicle's center of motion.

$$
d s^{2}=-d t^{2} \partial\left(x_{0}^{\prime}\right)^{2}+\sum_{1}^{3}\left(\left[\sigma^{a \mu}\left(t v_{s}\right)\left[1-\frac{2 G\left(N_{a 1} N_{a 2} m_{p 1} m_{p 2}\right)}{c^{2} r^{\prime}(.99)\left(N_{a 1} m_{p 1}+N_{a 2} m_{p 2}\right)}\right]^{-1}\right]\right)^{2} d t^{2} \partial\left(x_{\amalg}^{\prime}\right)^{2}
$$

More specifically, equation 5.18 describes the warping of space-time around the two particle accelerators generating gravitational forces $F_{g 1}^{\prime}$ and $F_{g 2}^{\prime}$ at a sub-light velocity $v_{s}$ as measured from the equilibrium coordinate $x_{0}$ (or center of mass $r_{c m}$ ) in the direction of travel. Therefore equation 5.18 describes the two gravity source warp field. In reference to Einstein's field equations, the motion at equilibrium coordinate $x_{0}$ is the movement along a geodesic to the curved surface of field function $\phi\left(x_{\amalg}^{\prime}(t)\right)$. The spatial velocity $v_{\text {rel }}$ as measured by a stationary observer outside of the curved space of the gravitational (or warp) fields generated by the two particle accelerators is given such that:

$$
v_{r e l}=\frac{x_{\longleftrightarrow}^{\prime}\left(a\left(r_{s}\right)\right)^{-1}}{t}=v_{S}\left[1-\frac{2 G\left(N_{a 1} N_{a 2} m_{p 1} m_{p 2}\right)}{c^{2} r^{\prime}(.99)\left(N_{a 1} m_{p 1}+N_{a 2} m_{p 2}\right)}\right]^{-1}
$$

The voltage values $V_{1}$ and $V_{2}$ (in the form of equation 2.11) required by the particle accelerators to accelerate particle mass values $m_{p 1}$ and $m_{p 2}$ to the verge of the speed of light thereby generating gravitational forces $F_{g 1}^{\prime}$ and $F_{g 2}^{\prime}$ and hence the two gravity source warp field is given such that:

$$
\begin{array}{ll}
V_{1}=-c\left[\left(\frac{m_{p 1} N_{1}}{q \Delta t}\right)^{2}-((.99) B)^{2}\right]^{\frac{1}{2}} ; & \left(\frac{m_{p 1} N_{1}}{q \Delta t}\right)^{2}>((.99) B)^{2} \\
V_{2}=-c\left[\left(\frac{m_{p 2} N_{2}}{q \Delta t}\right)^{2}-((.99) B)^{2}\right]^{\frac{1}{2}} ; & \left(\frac{m_{p 2} N_{2}}{q \Delta t}\right)^{2}>((.99) B)^{2}
\end{array}
$$

Where voltage values $V_{1}$ and $V_{2}$ vary in part according to acceleration numbers $N_{1}$ and $N_{2}$. The voltage value $V_{1}$ and $V_{2}$ correspond to gravitational forces $F_{g 1}^{\prime}$ and $F_{g 2}^{\prime}\left(V_{1} \rightarrow F_{g 1}^{\prime}\right.$ and $\left.V_{2} \rightarrow F_{g 2}^{\prime}\right)$, therefore the total $V_{\text {total }}$ required to generate the warp field is expressed such that:

$$
V_{\text {total }}=V_{1}+V_{2}
$$

Lastly, as shown in the previous section, proper time $t$ for a particle of mass $m_{0}$ is only experienced at the equilibrium point $x_{0}$ between the two gravitational forces $F_{g 1}^{\prime}$ and $F_{g 2}^{\prime}$ generated by the particle accelerators, hence where gravitational time dilation is zero as elucidated below.

$$
t_{0}=t \sqrt{1-\frac{(0)^{2}}{c^{2}}}=t \quad \rightarrow \quad v_{e s}=\sqrt{U_{g}^{\prime}\left(r_{1}, r_{2}\right)}=0
$$

However, the gravitational potential energy $U_{g}^{\prime}\left(r_{1}, r_{2}\right)$ acting on the particle of mass $m_{0}$ at position $x_{a}$ (such that: $x_{a} \neq x_{0}$ ) within the generated gravitational fields $F_{g 1}^{\prime}$ and $F_{g 2}^{\prime}$ will be equal to the sum (or net value) of integrals in respect to distances $r_{1}$ and $r_{2}$ as shown below (Young \& Freedman, 2004).

$$
U_{g}^{\prime}\left(r_{1}, r_{2}\right)=\int_{x_{i 1}}^{x_{a}}\left(\frac{G N_{a 1} m_{p} m_{0}}{\left(r_{1}\right)^{2}(.99)}\right) d r_{1}+\int_{x_{i 2}}^{x_{a}}\left(\frac{G N_{a 2} m_{p} m_{0}}{\left(r_{2}\right)^{2}(.99)}\right) d r_{2}
$$


This implies that every other position $x_{a}$ within the two gravity source warp field experiences a gravitational time slippage value of (where $v_{e s}=\sqrt{U_{g}^{\prime}\left(r_{1}, r_{2}\right)}$ of equation 4.07):

$$
t_{0}=t \sqrt{1-\frac{1}{c^{2}}\left(\int_{x_{i 1}}^{x_{a}}\left(\frac{G N_{a 1} m_{p} m_{0}}{\left(r_{1}\right)^{2}(.99)}\right) d r_{1}+\int_{x_{i 2}}^{x_{a}}\left(\frac{G N_{a 2} m_{p} m_{0}}{\left(r_{2}\right)^{2}(.99)}\right) d r_{2}\right)}
$$

Therefore, the limits of integration for both integrals of equations 5.26 and 5.27 range from initial points $x_{i 1}$ and $x_{i 2}$ at each particle accelerators to arbitrary a point $x_{a}$ within the warp field. Conclusively, a particle of mass $m_{0}$ within the warp field at any position $x_{a}$ that is not at the equilibrium point $x_{0}\left(x_{a} \neq x_{0}\right)$ between the two gravitational forces $F_{g 1}^{\prime}$ and $F_{g 2}^{\prime}$ are described by the inequality below.

$$
t>t \sqrt{1-\frac{1}{c^{2}}\left(\int_{x_{i 1}}^{x_{a}}\left(\frac{G N_{a 1} m_{p} m_{0}}{\left(r_{1}\right)^{2} .(99)}\right) d r_{1}+\int_{x_{i 2}}^{x_{a}}\left(\frac{G N_{a 2} m_{p} m_{0}}{\left(r_{2}\right)^{2}(.99)}\right) d r_{2}\right)}
$$

Or Alternatively:

$$
t>t_{0}
$$

An important consideration is that the two gravity source warp field also compresses space-time toward the vehicle in a direction opposite the direction of travel (which is opposite the direction of the velocity $v_{s}$ ) which would seem to compensate for the distance traveled through compressed space-time. The vehicle's velocity $v_{s}$ is measured from the equilibrium point $x_{0}$ between the particle accelerators generating gravitational forces $F_{g 1}^{\prime}$ and $F_{g 2}^{\prime}$, therefore the compression of space-time toward the vehicle in the direction opposite of travel will experience a slower rate of time or the time dilation of inequalities 5.28 and 5.29. Conclusively, the vehicle at equilibrium point $x_{0}$ between the particle accelerators generating gravitational forces $F_{g 1}^{\prime}$ and $F_{g 2}^{\prime}$ will travel faster through time (at a time-like displacement) in the direction of velocity $v_{s}$ as compared to the compressed space-time behind the vehicle "squeezing" space-time towards the vehicle in the direction opposite of travel in a space-like sense. As stated, space-time will return to its flat form once the warp field passes through the region of space-time.

\section{Conclusion}

The quantitative description of a hypothetical vehicle incorporating two particle accelerators which generate a two gravity source warp field while transiting through space-time is obtained by inserting values into key equations describing the warp field. Table 1 below gives the value of the gravitational constant $G$, the the speed of light $c$, the acceleration numbers $N_{a 1}$ and $N_{a 2}$ corresponding to both accelerators, the total mass values $m_{p 1}$ and $m_{p 2}$ of the charged particles (or electrons) in each particle accelerator, the value of the magnetic field $B$ in each accelerator, the sub-light velocity of the vehicle $v_{s}$, the charge of an individual electron $e$, the interval of time $\Delta t$ to which motion is measured, and the constant parameter of time $t_{a}$.

Table 1. Add a title here

\begin{tabular}{cc}
\hline$G$ & $6.673 \times 10^{-11} \mathrm{~N}^{\mathrm{m}} \mathrm{m}^{2} / \mathrm{kg}^{2}$ \\
\hline$c$ & $3.0 \times 10^{8} \mathrm{~m} / \mathrm{s}$ \\
$N_{a 1}$ & $2 \times 10^{25}$ \\
$N_{a 2}$ & $2 \times 10^{25}$ \\
$m_{p 1}$ & $600 \mathrm{~g}$ \\
$m_{p 2}$ & $600 \mathrm{~g}$ \\
$B$ & $1.26089 \times 10^{44} \mathrm{~T}$ \\
$v_{s}$ & $55,928.41 \mathrm{~m} / \mathrm{s}$ \\
$e$ & $1.60218 \times 10^{-19} \mathrm{C}$ \\
$\Delta t$ & $1 \mathrm{~s}$ \\
$t_{a}$ & $1 \mathrm{~s}$ \\
\hline
\end{tabular}

To expediently obtain a realistic value of a magnetic field for calculations, the value of magnetic field $B$ in table 1 is obtained using the magnetic force equation for accelerating an electron of charge $e$ as stated by Young and Freedman (2004) shown below.

$$
|\vec{F}|=|\vec{v} e \times \vec{B}|
$$

Where the magnitude of force value $|\vec{F}|$ and electron velocity vector $\vec{v}(v=.99 c)$ have values such that: 


$$
|\vec{F}|=\left(\frac{N_{a} c}{\Delta t}\right) m_{p} \quad ; \quad \vec{v}=\left(\begin{array}{lll}
0, & .99 c, \quad 0 \quad)
\end{array}\right.
$$

Notice that the force value $|\vec{F}|$ is equal to force $F_{a}$ of equation $2.0\left(|\vec{F}|=F_{a}\right)$ and the velocity component of the electron is an approximate 99 percent of the speed of light. The value of acceleration number $N_{a}$ is equal to the values of acceleration numbers $\quad N_{a 1}$ and $N_{a 2}\left(N_{a}=N_{a 1}=N_{a 2}\right)$ displayed in table 1. The mass $m_{p}$ is equal to the values of masses $m_{p 1}$ and $m_{p 2}\left(m_{p}=m_{p 1}=m_{p 2}\right)$ displayed in table 1. Each electron has an individual mass value of $9.109 \times 10^{-31} \mathrm{~kg}$ (or $9.109 \times 10^{-34} \mathrm{~g}$ ) (Young \& Freedman, 2004), however mass values $m_{p 1}$ and $m_{p 2}$ are massive clouds of electrons in two hypothetical giant particle accelerators. Hence, the forces of $|\vec{F}|$ and $F_{a}$ relate to pressure $P$ exerted on the cloud of particles and cross sectional chamber area $A$ of the particle accelerators such that (Young \& Freedman, 2004):

$$
|\vec{F}|=F_{a}=P A
$$

Therefore electromagnetic force $|\vec{F}|$ is acting on a massive density of electrons (up to $600 \mathrm{~g}$ in this example).

The values of table 1 are now applied to equations 5.14, 5.16, and 5.21 giving values of :

$$
\begin{gathered}
r^{\prime}=r_{a}+\frac{2 G\left(N_{a 1} N_{a 2} m_{1} m_{2}\right)}{c^{2}(.99)\left(N_{a 1} m_{1}+N_{a 2} m_{2}\right)}=55,937.4 \mathrm{~m} \\
a\left(r_{s}\right)=1-\frac{2 G\left(N_{a 1} N_{a 2} m_{1} m_{2}\right)}{c^{2} r^{\prime}(.99)\left(N_{a 1} m_{1}+N_{a 2} m_{2}\right)}=.99984 \\
v_{r e l}=v_{s}\left[1-\frac{2 G\left(N_{a 1} N_{a 2} m_{1} m_{2}\right)}{c^{2} r^{\prime}(.99)\left(N_{a 1} m_{1}+N_{a 2} m_{2}\right)}\right]^{-1}=55,937.4 \mathrm{~m} / \mathrm{s}
\end{gathered}
$$

Observe that the system velocity $v_{s}$ has an initial value of $55,928.4 \mathrm{~m} / \mathrm{s}$ (as shown in table 1), equation 6.5 shows that the warping of space-time of the two gravity source warp field produce an increased relative velocity $v_{\text {rel }}$ which is equal to $55,937.4 \mathrm{~m} / \mathrm{s}$. Therefore, the increase in velocity from system velocity $v_{s}$ to relative velocity $v_{r e l}$ is $9 \mathrm{~m} / \mathrm{s}$ which is very insignificant. To further illustrate this point, the values of table 1 are applied to the voltage (of equation 2.11) required by each hypothetical accelerator to generate a gravitational field a shown below

$$
\left|V_{a}\right|=\left|-c\left[\left(\frac{m_{p} N_{a}}{e \Delta t}\right)^{2}-((.99) B)^{2}\right]^{\frac{1}{2}}\right|=2.2469 \times 10^{55} \text { Volts }
$$

Both particle accelerators generating the two gravity warp field have the same values for their corresponding acceleration numbers $\quad N_{a 1}$ and $N_{a 2}\left(N_{a 1}=N_{a 2}=2 \times 10^{25}\right)$ as well total particle mass values $m_{p 1}$ and $m_{p 2}\left(m_{p 1}=m_{p 2}=600 \mathrm{~g}\right)$ as previously mentioned, thus the total value of voltage between both particle accelerators is given by the sum of voltage values $V_{\text {total }}$ (of equation 5.24) such that:

$$
\left|V_{\text {total }}\right|=\left|V_{1}+V_{2}\right|=4.4939 \times 10^{55} \text { Volts }
$$

One can conclude that the voltage required to utilize the two gravity source warp field for practical use is obscenely substantial and impractical to real world application as with the Alcubierre warp field (let along faster than light travel). The significance of the results of this paper is the elucidation of the theoretical possibility of generating a warp field of any magnitude with the use of available technology. Moreover, although the possibility of faster than light travel is seemingly distant, the results of this paper will hopefully represent continued research and experimentation to warp fields, which will inspire future advancements in the field.

\section{Acknowledgements}

I would like to acknowledge my lord and savior Jesus Christ, my mother Juanita Walker, my sister Brittani Shingles, my brother Kenneth J. Walker and Mr. Eugene Thomas for their support and guidance.

\section{References}

Anderson, D. L. (2016). Alcubierre warp drive. Retrieved from http://www.andersoninstitute.com/alcubierre -warp-drive.html 
Bench, A. T. (2016). Algebraic Derivation of the Schwarzschild Time Dilation Function. Retrieved from https://arxiv.org/ftp/physics/papers/0603/0603033.pdf

Cramer, J. G. (1996). The Alcubierre Warp drive. Analog Science Fiction \& Fact Magazine. Retrieved from https://www.npl.washington.edu/av/altvw81.html

Nipoti, C. (2013). Celestial Mechanics: lecture notes. University a di Bologna. Retrieved from http://campus.unibo.it/107918/1/celmech_2.pdf

Penrose, R. (2004). The road to reality: Comprehensive Guide to the laws of the Universe (pp. 222-223, 412, 413). New York, Alfred A. Knopf publishing.

Wald, R. M., (1984). General Relativity (pp. 17, 23-26, 31-38, 70,72,96, 101-104, 120-124). Chicago, ILL, Chicago Press, Ltd.

Walker, E. (2016). Gravitational Space-Time Curve Generation via Accelerated Charged Particles. Journal of Modern Physics, 7, 863-874. http://dx.doi.org/10.4236/jmp.2016.79078

Young, H. D., \& Freedman, A. R. (2004). Sears and Zemansky's University Physics (11th ed., pp. 444, 445, 517, 529, 530, 1431, 1450). San Francisco, Cal., Pearson, Addison Wesley.

\section{Appendix A}

Appendix A gives the formulation of the voltage value $V_{a}$ used in equation 2.11, 5.21, and 5.22, which is an excerpt from the Gravitational space-time curve generation via accelerated particles paper (Young \& Freedman, 2004; Walker, 2016).

It is of great importance to show the possibility and feasibility of accelerating a cloud of charged particles to an extent to where they actually produce a gravitational field in the real world (Walker, 2016). Thus, the Lorentz equation of electromagnetic force is applied to show this possibility. Lorentz force $F_{L}$ is as shown below (Walker, 2016).

$$
F_{L}=q[\bar{E}+\bar{v} \times \bar{B}]
$$

The velocity vector $\bar{v}$ is the velocity of each individual charged particle in the cloud density being accelerated by vector valued electromagnetic force $F_{L}$ (Walker, 2016). Where $q$ is the individual charge of each particle in the cloud density (Walker, 2016). The x-component of particle velocity vector $\bar{v}$ is given as approximated velocity value $v_{p}^{\prime}\left(v_{p}^{\prime}=.99 c\right)$ as shown below (Young \& Freedman, 2004; Walker, 2016).

$$
\bar{v}=\left(v_{p}^{\prime}, 0,0\right)=((.99 \times c), 0,0)
$$

The vector value for the magnetic field is given such that (Young \& Freedman, 2004; Walker, 2016):

$$
\bar{B}=(0, B, 0)
$$

The vector value for the electric field is given such that (Young \& Freedman, 2004; Walker, 2016):

$$
\bar{E}=(E, 0,0)
$$

Carrying out the cross product of velocity vector $\bar{v}$ and magnetic field vector $\bar{B}$ give the orthogonal vector value shown below (Young \& Freedman, 2004; Walker, 2016).

$$
\bar{v} \times \bar{B}=\left(0, \quad 0, v_{p}^{\prime} B\right)
$$

The value of Lorentz force vector $F_{L}$ at the given vector quantities of electric field $\bar{E}$, particle velocity $\bar{v}$, and magnetic field $\bar{B}$ are that of equation A.05 below (Young \& Freedman, 2004; Walker, 2016).

$$
F_{L}=q[\bar{E}+\bar{v} \times \bar{B}] \quad=\quad\left(q E, \quad 0, q v_{p}^{\prime} B\right)
$$

The magnitude of electromagnetic force vector $F_{L}\left(\left|F_{L}\right|\right)$ takes on a value such that (Walker, 2016):

$$
\left|F_{L}\right|=\left[(q E)^{2}+\left(q v_{p}^{\prime} B\right)^{2}\right]^{1 / 2}
$$

The magnitude of electromagnetic force $\left|F_{L}\right|$ is set equal to force $F_{a}$ corresponding to pressure $P_{0}$ and cross sectional area $A_{0}$ acting on the cloud or density of charged particles as shown in equation A.07 below (Young \& Freedman, 2004; Walker, 2016).

$$
\left|F_{L}\right|=F_{a}=A_{0} P_{0}
$$

Acceleration $a_{c}$ corresponds to the force $\left(F_{a}\right)$ per unit area $\left(A_{0}\right)$ acting on the cloud of accelerated charged particles, which correspond to pressure $P_{0}$ (where $F_{a}=A_{0} P_{0}$ ) (Walker, 2016). Recall that force $F_{a}$ takes on a value such that (Young \& Freedman, 2004; Walker, 2016): 


$$
F_{a}=a_{c} m_{p}=\left(\frac{N_{a} c}{\Delta t}\right) m_{p}
$$

The value of equation A.07 then becomes (Walker, 2016):

$$
\left|F_{L}\right|=\left(\frac{N_{a} c}{\Delta t}\right) m_{p}
$$

Equation A.09 can be expressed such that (Walker, 2016):

$$
\left(\frac{N_{a} c}{\Delta t}\right) m_{p}=q\left[(E)^{2}+\left(v_{p}^{\prime} B\right)^{2}\right]^{1 / 2}
$$

The task is to obtain the required voltage at a given acceleration number $N_{a}$, this will require one to solve equation A.10 for electric field $E$ as shown below (Walker, 2016).

$$
E=\left[\left(\frac{m_{p} N_{a} c}{q \Delta t}\right)^{2}-\left(v_{p}^{\prime} B\right)^{2}\right]^{1 / 2}
$$

Recall that velocity $v_{p}^{\prime}$ is the particles' approximate velocity at $99 \%$ of the speed of light (Walker, 2016). Thus, velocity $v_{p}^{\prime}$ is simply the product of the speed of light $c$ and the value $.99\left(v_{p}^{\prime}=(.99 \times c)\right)$ (Walker, 2016). The speed of light $c$ can then be distributed out of equation A.11, giving the value of equation A.12 such that (Walker, 2016):

$$
E=c\left[\left(\frac{m_{p} N_{a}}{q \Delta t}\right)^{2}-((.99) B)^{2}\right]^{1 / 2}
$$

The value of electrical field $E$ is equal to the negative partial derivative of voltage $V$ in respect to length $x$ (Young \& Freedman, 2004; Walker, 2016).

$$
E=-\frac{\partial V}{\partial x}
$$

Substituting this value (equation A.13) into equation A.12 gives the differential equation shown below (Young \& Freedman, 2004; Walker, 2016).

$$
\frac{\partial V}{\partial x}=-c\left[\left(\frac{m_{p} N_{a}}{q \Delta t}\right)^{2}-((.99) B)^{2}\right]^{1 / 2}
$$

This can be rearranged such that (Walker, 2016):

$$
\partial V=\left(-c\left[\left(\frac{m_{p} N_{a}}{q \Delta t}\right)^{2}-((.99) B)^{2}\right]^{\frac{1}{2}}\right) \partial x
$$

The corresponding integrals in respect to voltage $V$ and length $x$ are expressed such that (Walker, 2016):

$$
\int_{V_{i}=0}^{V_{f}=V_{a}} \partial V=\int_{x_{i}=0}^{x_{f}=\zeta}\left(-c\left[\left(\frac{m_{p} N_{a}}{q \Delta t}\right)^{2}-((.99) B)^{2}\right]^{\frac{1}{2}}\right) \partial x
$$

Where $\zeta$ is unit length, evaluating the integrals give the value of voltage $V_{a}$ such that (Walker, 2016):

$$
V_{a}=-c \zeta\left[\left(\frac{m_{p} N_{a}}{q \Delta t}\right)^{2}-((.99) B)^{2}\right]^{\frac{1}{2}}
$$

Length $\zeta$ is set to unity $(\zeta=1)$, therefore voltage $V_{a}$ can be expressed such that (Walker, 2016):

$$
V_{a}=-c\left[\left(\frac{m_{p} N_{a}}{q \Delta t}\right)^{2}-((.99) B)^{2}\right]^{\frac{1}{2}}
$$

Voltage $V_{a}$ is the product of electrical current $I$ and resistance $R\left(V_{a}=I R\right)$ (Young \& Freedman, 2004; Walker, 2016).

$$
I R=-c\left[\left(\frac{m_{p} N_{a}}{q \Delta t}\right)^{2}-((.99) B)^{2}\right]^{\frac{1}{2}}
$$

Equations A.18 and A.19 show the required voltage $V_{a}$ at acceleration number $N_{a}$ to produce gravitational force fields of charged particles at $99 \%$ if the speed of light (Walker, 2016). Thus the value of voltage $V_{a}$ or $I R$ 
sufficient to produce an acceleration that will generate gravity can be shown to exist in the real world with the condition of the inequality below (Walker, 2016).

$$
\left(\frac{m_{p} N_{a}}{q \Delta t}\right)^{2}>((.99) B)^{2}
$$

Voltage $V_{a}$ can be mapped to and corresponds to a gravitational force value $F_{g}$ at acceleration number $N_{a}$ as shown below (Walker, 2016).

$$
V_{a} \quad \rightarrow \quad F_{g}\left(N_{a}\right)
$$

Where gravitational force $F_{g}\left(N_{a}\right)$ is such that (Walker, 2016):

$$
F_{g}\left(N_{a}\right)=G \frac{\left(N_{a} m_{p}\right) m_{0}}{(.99)\left(\left|x_{\Perp}\right|^{2}\right)}
$$

This completes the formulation.

\section{Copyrights}

Copyright for this article is retained by the author(s), with first publication rights granted to the journal.

This is an open-access article distributed under the terms and conditions of the Creative Commons Attribution license (http://creativecommons.org/licenses/by/4.0/). 